\title{
A sequence of physical processes determined and quantified in large-amplitude oscillatory shear (LAOS): Application to theoretical nonlinear models
}

Simon A. Rogers, and M. Paul Lettinga

Citation: Journal of Rheology 56, 1 (2012); doi: 10.1122/1.3662962

View online: https://doi.org/10.1122/1.3662962

View Table of Contents: http://sor.scitation.org/toc/jor/56/1

Published by the The Society of Rheology

\section{Articles you may be interested in}

A sequence of physical processes determined and quantified in LAOS: Application to a yield stress fluid

Journal of Rheology 55, 435 (2011); 10.1122/1.3544591

New measures for characterizing nonlinear viscoelasticity in large amplitude oscillatory shear

Journal of Rheology 52, 1427 (2008); 10.1122/1.2970095

A geometrical interpretation of large amplitude oscillatory shear response Journal of Rheology 49, 747 (2005); 10.1122/1.1895801

A sequence of physical processes determined and quantified in LAOS: An instantaneous local 2D/3D approach

Journal of Rheology 56, 1129 (2012); 10.1122/1.4726083

Describing and prescribing the constitutive response of yield stress fluids using large amplitude oscillatory shear stress (LAOStress)

Journal of Rheology 57, 27 (2013); 10.1122/1.4754023

Large amplitude oscillatory shear (LAOS) measurements to obtain constitutive equation model parameters: Giesekus model of banding and nonbanding wormlike micelles Journal of Rheology 56, 333 (2012); 10.1122/1.3684751

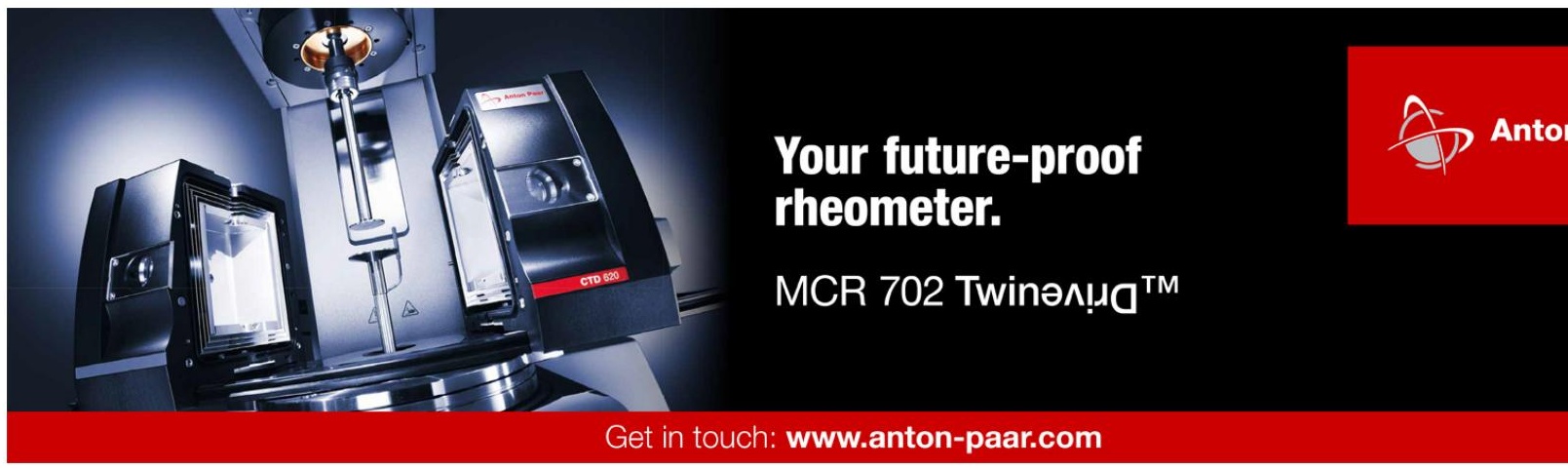




\title{
A sequence of physical processes determined and quantified in large-amplitude oscillatory shear (LAOS): Application to theoretical nonlinear models
}

\author{
Simon A. Rogers ${ }^{\mathrm{a})}$ and M. Paul Lettinga \\ Forschungszentrum Jülich GmbH, 52428 Jülich, Germany \\ (Received 24 March 2011; final revision received 18 September 2011; \\ published 29 November 2011)
}

\begin{abstract}
Synopsis
The nonlinear yielding responses of three theoretical models, including the Bingham, a modified Bingham, and Giesekus models, to large-amplitude oscillatory shear are investigated under the framework proposed recently by Rogers et al. (2011). Under this framework, basis states are allowed to wax and wane throughout an oscillation, an approach that conflicts directly with the assumptions of all Fourier-like linear algebraic approaches. More physical yielding descriptions of the nonlinear waveforms are attained by viewing the responses as representing purely elastic to purely viscous sequences of physical processes. These interpretations are compared with, and contrasted with, results obtained from linear algebraic analysis methods: Fourier-transform rheology; and the Chebyshev description of the so-called elastic and viscous stress components $\sigma^{\prime}$ and $\sigma^{\prime \prime}$. Further, we show that the discrepancies between the built-in model responses and parameters, and the interpretations of the Chebyshev and Fourier coefficients are directly related to misinterpretations of $\sigma^{\prime}$ and $\sigma^{\prime \prime}$ as being the elastic and viscous stress contributions. We extend these ideas and discuss how every linear algebraic analysis is likely to conflate information from predominantly elastic and viscous processes when a material yields. (C) 2012 The Society of Rheology. [DOI: $10.1122 / 1.3662962]$
\end{abstract}

\section{INTRODUCTION}

\section{A. LAOS in the literature}

Numerous methods exist for analyzing the response of materials to oscillatory shear which go beyond the classical determination of the storage and loss moduli at low strain amplitudes [Ferry (1980)]. These include Fourier transform (FT) rheology as introduced by Philippoff (1966), Harris and Bogie (1967), and Dodge and Krieger (1971), the use of dimensionless graphs [Cho et al. (2010)], the decomposition of the full stress response into parameters that have been called the elastic and viscous stress contributions via the stress decomposition (SD) [Cho et al. (2005) and enhanced by Yu et al. (2009)], and the description of these derived contributions by orthogonal Chebyshev polynomial series [Ewoldt et al. (2008)].

We argue that there are issues with each of these techniques that cannot be resolved, namely, that the Fourier and Chebyshev approaches rely on the erroneous assumption

\footnotetext{
a) Author to whom correspondence should be addressed; electronic mail: rogers.simon@gmail.com
} 
that material basis states are present throughout the entire waveform, and that the SD method assumes symmetries in nonlinear responses that are not present. We expose and investigate these shortcomings and present the beginnings of a resolution in this paper in the form of an alternative method of analysis, which takes a physical rather than mathematical approach.

Throughout this work, we shall frequently refer to "linear algebraic approaches." These are a general class of mathematically rigorous techniques for analyzing material responses to large-amplitude oscillatory shear (LAOS) that have their roots in the formalism of linear algebra. Each of the techniques assumes a basis set and describes the material responses from oscillatory shearing in terms of amplitudes (and possibly phases) of the basis states. In the classical analysis of linear regime responses, for instance, it is assumed that (only) elasticity and viscosity, the two fundamental responses of materials, occur simultaneously. In the language of linear algebra, one would say that, in the linear regime, the total material response is assumed to be the result of a linear combination of the two basis states; an elastic state and a viscous state. It is, therefore, reasonable that the analysis of linear-regime responses [Ferry (1980)] to find the dynamic moduli $G^{\prime}$ and $G^{\prime \prime}$, dynamic viscosities $\eta^{\prime}$ and $\eta^{\prime \prime}$, or modulus $G_{0}$ and phase difference (between input and output vectors) $\delta$ exhibits such assumptions; the elastic and viscous responses of the material are uncoupled and uniquely determined by finding the amplitudes of the corresponding basis states. For a sinusoidal application of strain, $\gamma(t)=\gamma_{0} \sin (\omega t)$, the elastic response is uniquely determined by the amplitude of the elastic basis state, or the inphase component, $\sin (\omega t)$, and the viscous response is uniquely determined by the amplitude of the viscous basis state, or the out-of-phase component, $\cos (\omega t)$. Using the amplitudes of the basis states, the full response can be reconstructed without loss of information. Reconstruction of a linear regime response can therefore be equated with understanding the relative contributions of elastic and viscous processes.

It is well established that in the nonlinear regime, where sinusoidal excitations result in nonsinusoidal outputs, the elastic and viscous parameters obtained using linear-regime assumptions and techniques are ill-defined. In this regard, it is clear that description of nonlinear responses requires the linear-regime analysis to be extended. In order to accurately reconstruct measured material responses, some researchers have taken the approach of extending the linear regime analysis in a linear algebraic way to encompass more basis states, without setting an upper limit on the number of basis states allowed. The basis states are commonly referred to as harmonics in analogy with audio tonal analysis. What these basis states/harmonics physically correspond to, beyond elastic and viscous states, has never been explained. This raises the questions as to whether extension to an infinite number of basis states is a physically meaningful approach to take, and why more basis states would be expected in the first place. We will argue here, by following the yielding responses of three nonlinear models, which materials can exhibit only two fundamental responses, those of elasticity and viscosity (which may themselves change with strain, stress, or their temporal derivatives). We propose that the way forward, in terms of understanding nonlinear responses to oscillatory shearing, is also by extending the traditional linear regime analysis, but not by applying ideas from linear algebra and infinitely expanding the size of the basis sets, or by changing the constituents of the basis, but by allowing the elasticity and viscosity, the two fundamental material responses, to independently wax and wane throughout an oscillation.

There are a number of reasons that make linear algebraic methods attractive to researchers studying nonlinear responses. They are mathematically rigorous, invertible, and convergent procedures that can be applied to any periodic response, regardless of shape. Furthermore, amplitudes (and phases if required) of basis states can be uniquely 
and quickly determined using well-established protocols, allowing for quick results to be obtained. By setting no upper bound on the number of basis states allowed, linear algebraic techniques can be used to obtain arbitrarily accurate reconstructions of any oscillatory stress response. However, we will argue that these benefits are outweighed, when analyzing yielding responses, by the shortcomings associated with the falsity of the assumptions that are often not discussed. This can be summarized by stating that reconstructions do not necessarily constitute useful physical descriptions.

FT rheology [Philippoff (1966), Harris and Bogie (1967), Dodge and Krieger (1971)] assumes periodicity in the time domain and transforms the response into the reciprocal frequency space. Usually in the literature, oscillatory strains are applied and the stress response is analyzed. A period of the total stress is then represented as a sum of sines and cosines, the frequencies of which are odd integer multiples of the excitation frequency, $\omega$. That is,

$$
\sigma\left(t ; \omega, \gamma_{0}\right)=\gamma_{0} \sum_{n}\left\{G_{n}^{\prime} \sin (n \omega t)+G^{\prime \prime}{ }_{n} \cos (n \omega t)\right\}
$$

where $n=2 k+1 ; \forall k \in \mathbf{N}$ (where $\mathbf{N}$ is the set of natural numbers, which includes zero) refers to the order of the harmonic and $G_{n}^{\prime}$ and $G^{\prime \prime}{ }_{n}$ are higher order generalized dynamic moduli. No upper bound exists for $n$, meaning an infinite number of basis states is assumed in FT-rheology. The stress is also often decomposed into a series of sine functions only, allowing for magnitude, $I_{n}$, and phase, $\delta_{n}$, information of the harmonics to be gathered. Thus, FT-rheology expresses a period of the total stress response in a linear algebraic manner, using the infinite set of sinusoids of different frequencies as a basis. In most cases, the amplitudes of the higher harmonics are presented normalized by the amplitude of the fundamental ( $n=1)$. Much work [Wilhelm (2002) and onwards] has gone into developing methods to reduce the experimental signal-to-noise ratio so that reliable measurements of higher harmonics can be made even when the relative intensities $I_{n} / I_{l}$ are as low as $1 \times 10^{-5}$. There has been much discussion about how to physically interpret the infinite number of basis states corresponding to $n \neq 1$ that could be used to describe stress responses to LAOS but no consensus has been reached. There has been comparatively little discussion on whether infinite extension of the size of the basis is a justifiable practice in the first place. Due to these problems, FT rheology has mainly been limited to engineering applications. Hyun and Wilhelm (2009) have recently suggested, in analogy with the third-order nonlinear susceptibility parameter $\chi^{(3)}$ from studies of nonlinear optics, that the magnitude of third Fourier harmonic divided by the square of the applied strain amplitude is a sensitive measure of polymer architecture in the medium-amplitude oscillatory shear (MAOS) regime (which is defined as the region where this quantity is constant. The MAOS regime for polymer melts typically extends between strain amplitudes of 10 and $200 \%$ ). The use of the third harmonic in other systems has mainly been restricted to determining the limits of the linear viscoelastic regime and checking experimental values against simulations. Most researchers present the Fourier frequency domain information of their material responses as individual harmonic magnitudes, often as functions of strain amplitude or frequency [Wilhelm (2002) and onwards], Renou et al. (2010)]. This practice, in conjunction with the assumptions and techniques for gathering information from linear-regime data, implicitly suggests a link between individual harmonics and distinct physical processes.

Many researchers have begun their investigations by displaying the material stress responses to LAOS as Lissajous-Bowditch curves [Philipoff (1966), Tee and Dealy (1975)]. In such representations, the full stress response is plotted parametrically against 
the oscillating strain (the so-called elastic representation) or the shear rate (the so-called viscous representation). A purely elastic stress response (a Hookean spring) when plotted as an elastic Lissajous-Bowditch curve forms a straight line, the gradient of which is equal to the elastic modulus, through the origin in the first and third quadrants, while a purely viscous stress response (a Newtonian fluid) forms an ellipse in the elastic representation, whose major and minor axes coincide with the stress and strain axes. In the viscous representation, the response of a Newtonian fluid forms a straight line, the gradient of which is equal to the viscosity. A general linear viscoelastic material response forms an ellipse in both representations, the major axis of which has an inclination to the positive strain axis between 0 and $\pi / 2$, an example of which is shown in the elastic representation in Fig. 1.

Cho et al. (2005) suggested a decomposition of stress responses to LAOS based upon constructions that use the elastic and viscous Lissajous-Bowditch curves as a starting point. The total stress response is decomposed into orthogonal contributions which are functions of strain and strain-rate. The interpretation comes about using similar assumptions of symmetry as FT rheology (that is, in the steady-state case, strain in one direction results in an identical response to strain in the opposite direction). In the SD method, the total stress is expressed as

$$
\sigma\left(\gamma, \frac{\dot{\gamma}}{\omega}\right)=\frac{\sigma\left(\gamma, \frac{\dot{\gamma}}{\omega}\right)-\sigma\left(-\gamma, \frac{\dot{\gamma}}{\omega}\right)}{2}+\frac{\sigma\left(\gamma, \frac{\dot{\gamma}}{\omega}\right)-\sigma\left(\gamma,-\frac{\dot{\gamma}}{\omega}\right)}{2},
$$

where the first term on the right-hand side is referred to as the "elastic stress," and the second term as the "viscous stress" because of their relative dependencies on the strain and strain-rate during an oscillatory test. These derived quantities are denoted $\sigma^{\prime}$ and $\sigma^{\prime \prime}$, respectively. In the SD method, the total stress is therefore the sum of these terms, $\sigma_{\text {total }}=\sigma^{\prime}+\sigma^{\prime \prime}$. We shall, in this work, only refer to them as $\sigma^{\prime}$ and $\sigma^{\prime \prime}$. A graphical way of forming the $\sigma^{\prime}$ and $\sigma^{\prime \prime}$ decompositions is to imagine a vertical line moving across the elastic or viscous Lissajous-Bowditch figure. At every point you wish to know the value of $\sigma^{\prime}$ or $\sigma^{\prime \prime}$, the line stops and a mark is made at the location that is halfway between the points where the stress orbit intercepts the line. In fact, using the previously mentioned

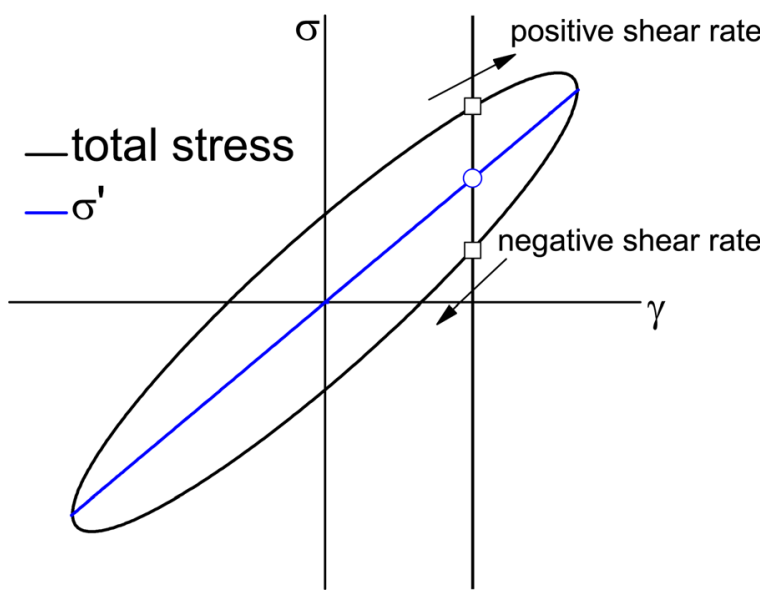

FIG. 1. The geometrical method of determining $\sigma^{\prime}$. The stress orbit intersects the vertical line at the square points, the centre of which, marked by a circle, represents the value of $\sigma^{\prime}$ at that strain. 
assumption about the symmetry of the stress response, expressed in the same notation as used in Cho et al. (2005), $\sigma\left(-\gamma, \frac{\dot{\gamma}}{\omega}\right)=-\sigma\left(\gamma,-\frac{\dot{\gamma}}{\omega}\right)$ (that is, the Lissajous figures are assumed to show rotational symmetry of order 2 about the origin), it is possible to rearrange the original definition of the stress decomposition to read

$$
\sigma\left(\gamma, \frac{\dot{\gamma}}{\omega}\right)=\frac{\sigma\left(\gamma, \frac{\dot{\gamma}}{\omega}\right)+\sigma\left(\gamma,-\frac{\dot{\gamma}}{\omega}\right)}{2}+\frac{\sigma\left(\gamma, \frac{\dot{\gamma}}{\omega}\right)+\sigma\left(-\gamma, \frac{\dot{\gamma}}{\omega}\right)}{2},
$$

which makes this interpretation clearer. In the SD method, Eqs. (2) and (3) are equivalent. We show in Fig. 1 a visual guide to finding $\sigma^{\prime}$ using as an example a (constructed) typical linear viscoelastic response. The solid black ellipse shows the total stress response, while the blue line represents $\sigma^{\prime}$. The vertical black line (which is to be interpreted as having moved across the figure) is intersected by the stress orbit at the points marked by squares. The section of the vertical line between the two squares has a centre at the circle. The point of the circle marks the value of $\sigma^{\prime}$ at that particular strain.

A similar process with a viscous Lissajous-Bowditch curve (where the stress is plotted as a parametric function of the rate) yields $\sigma^{\prime \prime}$. Therefore [Eq. (3)], $\sigma^{\prime}$ is an average of the stress responses of a system at equal (global) strains from shear rates of equal magnitude but opposing signs. Likewise, $\sigma^{\prime \prime}$ is an average of the stress responses of the same system at equal shear rates from (global) strains of equal magnitude but opposing signs. This is a crucial point that will be important to keep in mind throughout this work.

If one takes more than a cursory look at the SD definitions presented in Eqs. (2) and (3), it becomes apparent that SD requires only half of a complete waveform to compute $\sigma^{\prime}$ and $\sigma^{\prime \prime}$. The first term on the right hand side of Eq. (2) and the second term on the right hand side of Eq. (3) differ only in the sign between the two terms of the denominator; from Eq. (2) $\sigma^{\prime}$ is equal to half the difference between stresses at opposite (global) strains and equal shear rates, while from Eq. (3) $\sigma^{\prime \prime}$ is half the sum of the stresses from the same two points. This means that only positive (or negative) shear rate information [the top (bottom) half of the Lissajous figure displayed in Fig. 1] needs to be retained in order to perform the SD. Using the second term on the right hand side in Eq. (2) and the first term on the right of Eq. (3) show how the SD can be performed with only the positive (or negative) strain information. The SD method, therefore, makes the implicit assumption that based on the symmetries present [Eqs. (2) and (3)], the elastic and viscous stress contributions, when presented as functions of time, constitute $2^{*} \infty$ (spinning sidle) frieze groups. Nonlinear stress responses, in general, constitute $\infty \times$ (step) frieze groups [Rogers and Vlassopoulos (2010)], while linear stress responses constitute $2 * \infty$ (spinning sidle) frieze groups. We will show that the symmetry assumptions placed on $\sigma^{\prime}$ and $\sigma^{\prime \prime}$ are too strict for nonlinear responses and that they lead to conflation of information from elastic and viscous processes.

The SD method changed the problem of describing nonlinear waveforms from having to describe two open shapes (the elastic and viscous Lissajous-Bowditch curves) to only having to describe two lines $\left(\sigma^{\prime}\right.$ and $\left.\sigma^{\prime \prime}\right)$.

Ewoldt et al. (2008) noted that $\sigma^{\prime}$ and $\sigma^{\prime \prime}$ are related directly to the Fourier decomposition by

$$
\sigma^{\prime} \equiv \gamma_{0} \sum_{n} G_{n}^{\prime}\left(\omega, \gamma_{0}\right) \sin (n \omega t), \sigma^{\prime \prime} \equiv \gamma_{0} \sum_{n} G^{\prime \prime}{ }_{n}\left(\omega, \gamma_{0}\right) \cos (n \omega t)
$$

where $n=2 k+1 ; \forall k \in \mathbf{N}$, as in the formalism of FT rheology. The relations in Eq. (4) are another example of a description using linear algebraic ideas: $\sigma^{\prime}$ is expressed in terms 
of the orthogonal set of sines of different frequencies and $\sigma^{\prime \prime}$ is expressed in terms of the orthogonal set of cosines of different frequencies. The amplitudes of the various basis states are referred to as higher-order (dynamic) moduli. Ewoldt et al. (2008) proposed a further level of orthogonal decomposition by describing $\sigma^{\prime}$ and $\sigma^{\prime \prime}$ as series of Chebyshev polynomials of the first kind, $T_{\mathrm{n}}$, which are defined by the recurrence relation

$$
\begin{aligned}
T_{0}(x) & =1 \\
T_{1}(x) & =x, \\
T_{n+1}(x) & =2 x T_{n}(x)-T_{n-1}(x) .
\end{aligned}
$$

These polynomials form an orthonormal basis set over the interval $[-1,1]$, with respect to the inner product $\frac{2}{\pi} \int_{-1}^{1} T_{n}(x) T_{m}(x) \frac{d x}{\sqrt{1-x^{2}}}$ (except for the case where $n$ and $m=0$ when the prefactor becomes $\frac{1}{\pi}$ ) and can be used to unambiguously and uniquely describe $\sigma^{\prime}$ and $\sigma^{\prime \prime}$ by

$$
\begin{gathered}
\sigma^{\prime}(\gamma)=\gamma_{0} \sum_{n:} e_{n}\left(\omega, \gamma_{0}\right) T_{n}(\gamma), \\
\sigma^{\prime \prime}\left(\frac{\dot{\gamma}}{\omega}\right)=\dot{\gamma}_{0} \sum_{n:} v_{n}\left(\omega, \gamma_{0}\right) T_{n}\left(\frac{\dot{\gamma}}{\omega}\right),
\end{gathered}
$$

where $n=2 k+1 ; \forall k \in \mathbf{N}$ and the $e_{n}$ 's and $v_{n}$ 's, the amplitudes of the basis states, are the elastic and viscous (Chebyshev) coefficients of order $n$. The linear algebraic relations in Eq. (6) express $\sigma^{\prime}$ and $\sigma^{\prime \prime}$ in terms of the basis of orthonormal Chebyshev polynomials. The relations expressed in Eqs. (4) and (6) detail how the Chebyshev approach is related to FT rheology. One of the most exciting and enticing reasons for using Chebyshev polynomials of the first kind of order $n$ is that under a limited range of conditions for $n=1$ and 3, physical interpretations can be attached to both the $e_{n}$ 's and $v_{n}$ 's. $e_{1}$ and $v_{1}$ are interpreted as describing the linear elastic and viscous responses. When $e_{n}<e_{3}<e_{1}$ for all values of $n \geq 5$, that is, when the third order harmonic is the leading order nonlinearity, positive values of $e_{3}$ result in larger stresses at larger strains and so are considered to describe strain-hardening. Similar arguments lead to the interpretation of negative values of $e_{3}$ as depicting strain-softening and $v_{3}$ as describing the shear-thickening/shear-thinning behavior. The physical interpretations attached to the first and third-order Chebyshev coefficients also rely on the implicit assumption (from the intimately related FT rheology) made by many researchers that, as in the linear regime, individual harmonics should be related to separate physical processes or behaviors. For the first time, terms such as (intracycle) strain-hardening $\left(e_{3}>1\right)$, strain-softening $\left(e_{3}<1\right)$, shear-thickening $\left(v_{3}>1\right)$, and shear-thinning $\left(v_{3}<1\right)$ could be quantified. Indeed, quantification is not required for a "first-glance" analysis under this approach, as these behaviors can simply be read off the elastic and viscous Lissajous-Bowditch curves by following $\sigma^{\prime}$ and $\sigma^{\prime \prime}$ as both strain and shear rate increase. The Chebyshev series description of $\sigma^{\prime}$ and $\sigma^{\prime \prime}$, like FT rheology, suffers from a potential problem of large numbers of higher harmonics in LAOS measurements. Many material responses to LAOS show a number of harmonics well in excess of the first two for which physical interpretations have been proposed. The limiting factor to the number of higher harmonics that can be measured is often only the precision of the measuring setup and the inherent signal-to-noise ratio.

The important underlying assumption that comes from their linear algebra roots that links FT rheology and the Chebyshev description of $\sigma^{\prime}$ and $\sigma^{\prime \prime}$ is that, as in the linear regime, the total stress response is best represented by a linear combination of basis states. Put another way, these commonly used analysis methods both make the implicit 
assumption that the basis states (the assumed underlying "true" responses of the systems under investigation) are present in linear combination with one another, or absent, throughout the total stress response. As a result, both methods have an inherent weakness in that descriptions of responses that are transient, in terms of alternating between states, on the timescale of one period are misrepresented because of false assumptions. Using these formalisms, the problem of describing the total stress response is reduced to calculating the amplitudes (and possibly phases) of the various basis states present. FT rheology assumes the infinite set of trigonometric functions [Eq. (1)] as a basis and lacks physical interpretability for all harmonics higher than the fundamental. The Chebyshev description of the SD constructions $\sigma^{\prime}$ and $\sigma^{\prime \prime}$ uses the infinite set of Chebyshev polynomials of the first kind as a basis and has physical interpretations for only the first and third harmonics under limited conditions [Eq. (6)]. There are currently no published reports of an infinite orthonormal basis set that can be used to describe material responses in a physically meaningful way.

A number of contributions in recent years have suggested that physically meaningful interpretations can be garnered not from individual harmonics, but rather from considering groups of (Fourier or Chebyshev) harmonics. Klein et al. (2007) also used the ideas of linear algebra by suggesting that nonlinear material responses could be described by linear combinations of sine, rectangular, triangular, and sawtooth waves. With the exception of the sine wave, each of these wave-types, viewed in the frequency domain, contain infinitely many higher harmonics of differing amplitudes and phases. Klein et al. attached to each wave a physical interpretation; sine waves represented linear responses, rectangular waves were attributed to strain softening, triangular waves represented strain hardening, and a sawtooth contribution was attributed to the presence of shear bands or wall slip.

As well as introducing the Chebyshev description of $\sigma^{\prime}$ and $\sigma^{\prime \prime}$, including the interpretations of the (individual) first and third harmonics, Ewoldt et al. (2008) also defined a number of meaningful viscoelastic moduli and dynamic viscosities based on the geometry of a nonlinear stress response. These moduli and viscosities can be calculated from the Chebyshev (or Fourier) coefficients and reduce to $G^{\prime}$ and $\eta^{\prime}$, respectively, in the linear regime. In order to accurately calculate the local measures suggested by Ewoldt et al., an experimentalist has two options: calculate the values from the harmonic series representation, using the same number of harmonics that are assumed to accurately reconstruct the stress response; or calculate the moduli and viscosities directly from the total stress responses and their derivatives. It should be noted that the series description for the suggested moduli and viscosities include the first and third order harmonics, which have their own (sometimes conflicting) suggested interpretations.

Most recently, Rogers et al. (2011) suggested that nonlinear stress responses are best viewed as representing a sequence of physical processes. This was the first suggestion of a technique not based on the assumptions of linear algebra to analyzing LAOS responses. Rogers et al. (2011) also showed that power-law fluid responses with indices less than unity contain infinitely many higher harmonics and linked this to the assumption of interpreting individual harmonics as relating to separate physical processes. Higher harmonics, in this case, are inseparable and therefore ought not to be viewed as representing separate physical processes or behaviors.

\section{B. Motivation and manuscript layout}

The sequence of physical processes (SPP) work of Rogers et al. (2011) motivated us to use two models that are known to approximate the nonlinear yielding behavior of a large class of complex fluids to examine more closely the interpretations of the 
different analysis techniques described above. In all cases presented here, the applied oscillatory shear field is assumed to be sinusoidal, so that the time-dependent shear rate is identically out of phase with the strain. In addition, we analyze a special case of a toy model which can be viewed as approximating the response of colloidal glasses. Investigations using these three examples suggest that even if an orthonormal basis set could be found which allowed physical interpretations (larger stresses at larger strains, for instance), that those interpretations would be, at best incomplete, and at worst, fundamentally inaccurate for a large class of material responses. We further suggest that, despite the mathematical rigor with which they are defined, the SD parameters $\sigma^{\prime}$ and $\sigma^{\prime \prime}$ do not, and cannot, accurately depict the true elastic and viscous stresses of a system undergoing yielding while being subjected oscillatory shear for the models we studied and thus for a wide range of materials. We evolve these ideas toward their logical conclusion and state that any linear algebraic approach to describing yielding responses to LAOS, no matter how rigorously defined, will potentially conflate information from elastic and viscous processes.

The methods we use to analyze yielding responses in this article do not readily lend themselves to the construction of a standard LAOS recipe in the way that linear algebraic techniques do. Interested readers are directed to the earlier work of Rogers et al. (2011) for a full phenomenological treatment of the SPP technique.

The first two examples given will be theoretical yield-stress materials, based on the Bingham model. The first example is the Bingham model as proposed by Yoshimura and Prud'homme (1987) which behaves perfectly Hookean with no dissipation until a yield stress is exceeded, whereby the model responds by flowing, with the stress from flowing being proportional to the shear rate. Flow ceases in the Bingham model when the stress drops to the yield stress again, the point beyond which the model response is elastic. The arguments presented in this section can be easily expanded to encompass the response of the Herschel-Bulkley model (where a power-law index that is not necessarily equal to unity is included in the flow term) also. In the second example, we modify the Bingham model so that the model continues to flow until the rate is instantaneously zero. That is, we include a nonzero static yield stress, but design the dynamic yield stress to be zero (the modification to the Bingham model, which has identical static and dynamic yield stresses). This can be thought of as approximating a physical (perhaps colloidal) system that has some sort of structure that is completely destroyed once the yield conditions are exceeded. The structure then reforms the instant the material comes to rest at the point of zero instantaneous rate. This is a toy model and will only be used at a specific frequency and amplitude for ease of use. The third example given is the Giesekus model [Bird et al. (1987)] which has been used to model nonlinear oscillatory and steady-shear flow of a wide variety of materials. To conclude, we present arguments as to why the responses seen in each scenario can be viewed as being representative of nonlinear yielding responses in general.

\section{RESULTS AND DISCUSSION}

\section{A. The Bingham model}

The Bingham model, as expressed by Yoshimura and Prud'homme (1987), is

$$
\begin{array}{ll}
\tau=G \gamma_{E} & \left(\left|\gamma_{E}\right|<\gamma_{y}\right), \\
\tau=G \gamma_{E}+\mu \dot{\gamma} & \left(\left|\gamma_{E}\right|=\gamma_{y}\right),
\end{array}
$$

where $\tau$ designates the stress, $G$ the elastic modulus, $\mu$ the Bingham (plastic) viscosity, $\gamma_{E}$ the elastic (recoverable) strain, $\dot{\gamma}$ the strain rate, and $\gamma_{y}$ the yield strain. The yield stress, 
$\tau_{y}$, is intimately related to the yield strain by $\tau_{y}=G \gamma_{y}$. At stresses/strains smaller than the yield conditions, the model behaves elastically and dissipates no energy; the stress is in phase with the strain. If the applied stress/strain exceeds the yield condition, the stress increases proportional to the shear rate. The stress in the viscous regime is completely out of phase with the strain. The Bingham model can be thought of as a special case of the Herschel-Bulkley model [Herschel and Bulkley (1926)] which includes a power-law exponent of the shear rate in Eq. (7). In the Bingham model, the power-law exponent is simply unity. We shall preferentially use the Bingham model but note that the comments of this section relate also to the response of the more general Herschel-Bulkley model. A representative stress response of the Bingham model, with unit modulus, viscosity, and yield stress, to oscillatory shear of strain amplitude ten and frequency one is shown in Fig. 2 as black lines. While the jump in stress observed under these conditions at yielding is perhaps unphysical, suggesting that the conditions imposed are outside those intended for use, the general shape of the Bingham model is well captured: regions of elastic extension and viscous flow are clearly identifiable. The decomposed functions $\sigma^{\prime}$ and $\sigma^{\prime \prime}$ are shown as blue and red lines, respectively. It should be noted that the stress orbits clockwise in the elastic representation and anticlockwise in the viscous.

A cursory examination of $\sigma^{\prime}$, shown in blue in the elastic representation in Fig. 2, reveals that at a global strain of zero, $\gamma=0, \sigma^{\prime}$ is zero. As the applied deformation is increased, $\sigma^{\prime}$ remains zero until the strain is equal to $\gamma_{0}-2 \gamma_{\text {yield. }}$. At this point, $\sigma^{\prime}$ jumps to a positive value, which decreases with further increases of strain. This sudden increase in $\sigma^{\prime}$ followed in sequence by the decreasing response of $\sigma^{\prime}$ with increasing strain would be interpreted as showing a short interval of strain-hardening followed by strain-softening. However, by definition, all strain-related stress changes in the Bingham model are linear. The interpretations of the decomposed functions from the SD method are clearly at odds with design of the model.

Describing $\sigma^{\prime}$ and $\sigma^{\prime \prime}$ in terms of a Chebyshev series gives indications of the generalized intracycle behaviors as presented by Ewoldt et al. (2008). Using this analysis for the current case, we obtain a positive first-order elastic coefficient $\left(e_{1}=0.17\right)$, and a positive normalized third-order elastic coefficient $\left(e_{3} / e_{1}=0.40\right)$. A positive first-order viscous coefficient $\left(v_{1}=1.06\right)$ and a positive normalized third-order viscous coefficient $\left(v_{3} / v_{1}=0.09\right)$ are also acquired. Ewoldt et al. (2008) suggest that this should be interpreted as showing (intracycle) strain hardening $\left(e_{3} / e_{1}>0\right)$ and shear thickening $\left(v_{3} / v_{1}>0\right)$. These interpretations also conflict with the designed model properties.

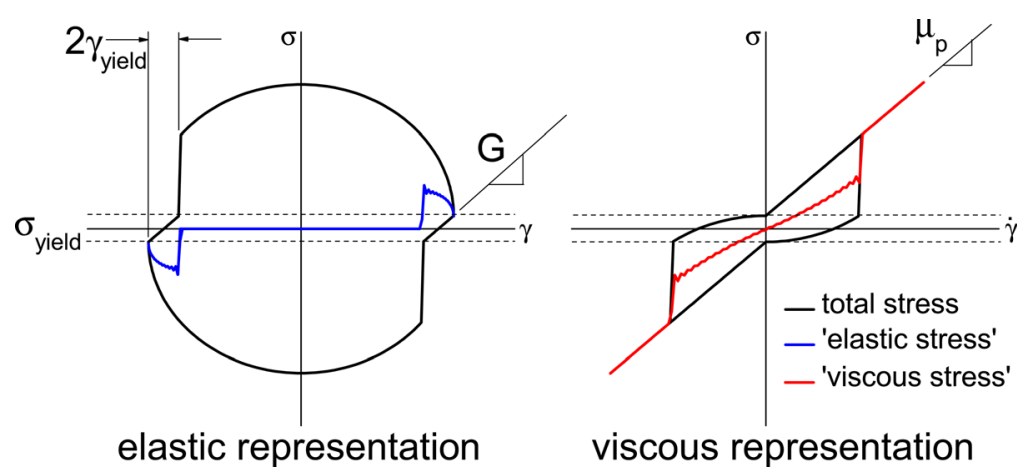

FIG. 2. A representative stress response of the Bingham model with relevant quantities from the SD method shown. 
An alternative way to view and analyze nonlinear response curves, such as those displayed in Fig. 2, which does not rely on the assumptions of linear algebra, is as a SPP, as suggested by Rogers et al. (2011). In this framework, the stress response is not decomposed into a linear sum of contributions (which are assumed to exhibit particular strict symmetries) that are dependent on strain and strain-rate as in the SD method, but rather viewed as being the result of a sequence of distinct physical processes. The Bingham model is somewhat trivial to analyze in this framework because it is created piecewise to begin with by demanding that a viscous response follows an elastic one. Nevertheless, it is a worthwhile exercise applying these ideas to the typical Bingham response to LAOS displayed in Fig. 2 because of the relative ease with which the model parameters are determined. We remind the reader at this point that perfectly elastic (Hookean) extension appears in the elastic representation of the Lissajous-Bowditch curve as a straight line, the gradient of which is equal to the modulus, while perfectly viscous (Newtonian) flow appears in the viscous representation of the Lissajous-Bowditch curve as a straight line, the gradient of which is equal to the viscosity.

Beginning at zero stress (near $-\gamma_{0}$ ) and observing the response to increasing strain (reading the Lissajous-Bowditch figure from left to right), the stress increases linearly with strain proportional to the modulus, $G$, as depicted by the portions of the Lissajous curve which are straight in the elastic representation in Fig. 2. Once the yield stress, shown by the dashed lines in Fig. 2, is exceeded, the Bingham material flows with a plastic viscosity $\mu_{p}$ which is seen in the linearity of that portion of the stress trajectory in the viscous representation. Therefore, using the SPP approach, we can completely reconstruct the Bingham constitutive equation from half a single oscillatory response. While this may seem like a simple task given the nature of the model, the best the FT and Chebyshev approaches can do is to describe a single particular response curve with potentially an infinite number of physically meaningless parameters. It should also be noted that the large number of parameters generated by the FT and Chebyshev approaches to describe the response of the Bingham model [see, for instance, Klein et al. (2007)] will change with each strain amplitude and frequency investigated (as long as the yield conditions are exceeded), while the parameters obtained using the SPP approach will remain the same.

It can be said that the Bingham model displays two fundamental responses-purely Hookean elastic extension for all stresses smaller than the yield stress and Newtonian viscous flow for all stresses above the yield stress. It is of primary importance to note that the viscous response of the Bingham model is not present continuously throughout the waveform, but rather comes into existence following yielding. In order to describe the Newtonian region of the Bingham model, a linear algebraic approach necessarily takes into account the (unrelated) elastic extension at the start of a cycle. It follows that the viscous response of the Bingham fluid cannot be represented by any linear algebraic analysis technique, because of their inherent assumption of the symmetries exhibited by the basis states (the sinusoidal basis states of order $n$ assumed by FT-rheology are equal to zero $2 n+1$ times every period and are antisymmetric about those points). It is also significant that neither this simple sequence of physical processes (elastic extension which gives way to viscous flow) nor the values of the modulus or yield stress can be captured in any linear algebraic analysis. It should be noted that the Bingham viscosity can be calculated from a linear algebraic approach. Ewoldt et al. (2008) define the large-rate dynamic viscosity $\eta_{L}^{\prime} \equiv \sigma /\left.\dot{\gamma}\right|_{\dot{\gamma}=\dot{\gamma}_{0}}=(1 / \omega) \sum_{n} G_{n}^{\prime \prime}=v_{1}+v_{3}+\ldots$ This parameter does equal the plastic viscosity, $\mu_{p}$, included in the model, but can only be calculated at the point of maximum shear rate. In the more general Herschel-Bulkley case, the fluid response of the system is described by a power-law which results in a shear rate dependence of the 
viscosity. The large-rate dynamic viscosity is only a measure of the material viscosity at a single point, and therefore contains no information regarding the rate dependence of the viscosity. A series of LAOS tests, of varying shear rate amplitudes, would need to be carried out using the large-rate dynamic viscosity in order to provide the same amount of information as one oscillation viewing the response in the SPP framework.

The Chebyshev description of $\sigma^{\prime}$ and $\sigma^{\prime \prime}$ and FT rheology both fail to correctly describe the physics for similar reasons described above. This is an important point which we shall find ourselves coming back to throughout this work: when the fundamental material responses are not always present, linear algebraic methods fail to clearly and fully describe the physics. It is clear that some linear algebraic methods such as FT rheology [which was applied to the Bingham model in Klein et al. (2007)] and the Chebyshev approach are convergent, so that it is possible to reconstruct with arbitrary accuracy any periodic response function. However, reconstructions do not necessarily constitute useful physical descriptions, especially when the fundamental assumptions leading to the reconstruction are false. What is less clear, and what has not been discussed fully in the literature up to now, is how these linear algebraic methods cope with responses that are not simply linear superpositions of a basis.

\section{B. The modified Bingham model}

We next modify the Bingham model so that ideal Hookean elastic behavior is followed in sequence by ideal Newtonian flow. In this toy model there thus exists a nonzero static yield stress, but once yielded, the model behaves Newtonianly until the instantaneous rate returns to zero. The stress response of this modified Bingham material is shown in Fig. 3 in black along with $\sigma^{\prime}$ in blue and $\sigma^{\prime \prime}$ in red. The quantities are plotted in elastic (left) and viscous (right) Lissajous-Bowditch figures.

Similar comments can be made regarding the form of $\sigma^{\prime}$ in the modified Bingham model as in the standard Bingham model: $\sigma^{\prime}$ is zero at zero strain and remains so until the strain is increased to $\gamma_{0}-\gamma_{\text {yield }}$. At this point of strain, $\sigma^{\prime}$ begins to increase, reaches a maximum and then returns to zero again when the strain is equal to $\gamma_{0}$. It is worth noting that in the region where the designed elastic response of the system is causing the stress to change linearly with strain, $\sigma^{\prime}$ is nonlinear and has a sign opposite to that of the stress. It is also worth noting that during this part of the waveform, where there is, by design, no dissipation of energy, $\sigma^{\prime \prime}$ is nonzero. Taking the SD and Chebyshev extension approach, we would interpret this as an interval of strain hardening followed by an interval of

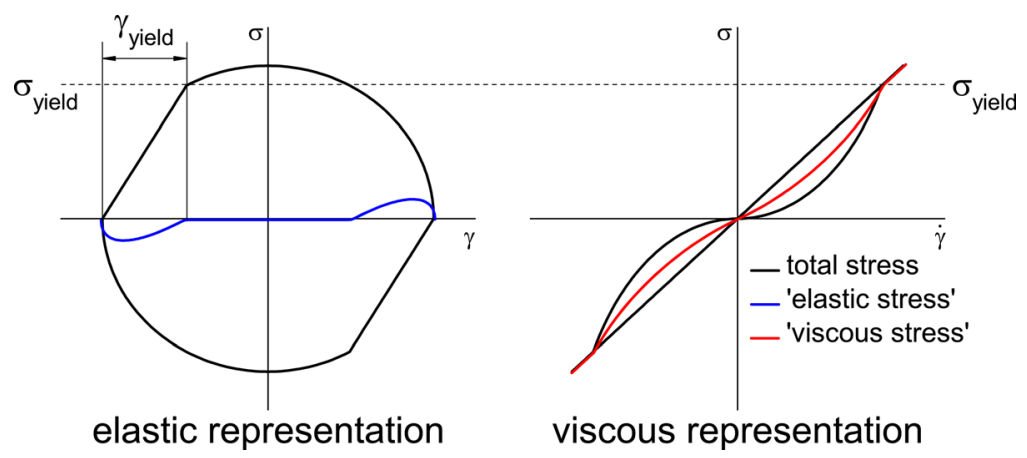

FIG. 3. The stress response of our modified Bingham model with relevant quantities from the SD method shown. 
strain softening, an interpretation that is clearly at odds with the way the model is designed.

Similar remarks can be made about $\sigma^{\prime \prime}$, shown in the viscous representation in Fig. 3. Beginning at zero and increasing the shear rate, an interval of shear thickening is followed by a small region of Newtonian flow. The reason we are able to discern a small section of Newtonian flow is that the yield stress is smaller than the stress amplitude achieved by the material under the flow conditions imposed. It can be seen from Fig. 3 that above the dotted line marking the yield condition the stress response is symmetric about zero strain and maximum shear rate. The interpretation of $\sigma^{\prime \prime}$ with increasing strain as representing shear thickening is in conflict with the design of the model.

Using the methods outlined by Ewoldt et al. (2008) we can ascribe numbers to the generalized intracycle responses of the modified Bingham response. We measure a positive first-order elastic coefficient and a negative third-order intracycle strain softening coefficient $\left(e_{3} / e_{1}<0\right)$. Further, a positive first-order viscous coefficient and a positive third-order shear thickening coefficient $\left(v_{3} / v_{1}>0\right)$ are calculated. These terms are in obvious conflict with the design of the model.

Using the SPP framework, the response we designed into our model is immediately obvious. Starting at zero stress in the elastic representation at $-\gamma_{0}$, the stress increases linearly with increasing strain, indicative of a purely elastic response, until the yield conditions are exceeded whereby the material flows in a Newtonian manner. The elastic response of the system causes the corresponding stress portion of the waveform to appear as a straight line when plotted against strain and the viscous response of the material, postyielding, appears as a straight line when plotted against rate.

In the modified Bingham model, as in the standard version, the viscous state, which is defined by a single value of viscosity, is not present throughout an entire oscillation but only comes into existence once the yield stress has been exceeded. It is, therefore, not possible to describe the viscous response of this model with a single basis state in any linear algebraic analysis technique. Similar to the standard Bingham model, the large rate dynamic viscosity as defined by Ewoldt et al. (2008) does correctly reproduce the viscosity of this model. It does so, however, only at the point of maximum rate.

\section{The Giesekus model}

The Giesekus model, as presented by Bird et al. (1987), is given by

$$
\begin{gathered}
\boldsymbol{\sigma}=\boldsymbol{\sigma}_{s}+\boldsymbol{\sigma}_{p}, \\
\boldsymbol{\sigma}_{s}=\eta_{s} \dot{\gamma}, \\
\boldsymbol{\sigma}_{p}+\lambda_{1} \boldsymbol{\sigma}_{p(1)}+\alpha \frac{\lambda_{1}}{\eta_{p}}\left\{\boldsymbol{\sigma}_{p} \cdot \boldsymbol{\sigma}_{p}\right\}=\eta_{p} \dot{\gamma},
\end{gathered}
$$

where $\boldsymbol{\sigma}_{s}$ is the stress tensor for the solvent, $\boldsymbol{\sigma}_{p}$ is the polymer stress tensor, $\boldsymbol{\sigma}_{p(1)}$ is the upper convected time derivative of the polymer stress, $\eta_{s}$ is the solvent viscosity, $\eta_{p}$ is the polymer viscosity, $\lambda_{1}$ is the relaxation time, and $\alpha$ is the so-called mobility factor. In the linear viscoelastic regime, the Giesekus model reduces to the linear Jeffreys model which can be mechanically approximated by a Maxwell element (a spring and dashpot in series) in series with another dashpot.

The stress response of the Giesekus model to sinusoidal strain $\gamma(t)=\gamma_{o} \sin (\omega t)$ was calculated with the parameters $\lambda_{1}=1 \mathrm{~s}, \eta_{s}=0.01 \mathrm{~Pa} \cdot \mathrm{s}, \eta_{p}=10 \mathrm{~Pa} \cdot \mathrm{s}$, and $\alpha=0.3$. This choice of parameters, chosen for the comparison available to data published by Ewoldt et al. (2008), gives rise to the derived quantities of the zero-shear-rate viscosity 
$\eta_{0}=\eta_{s}+\eta_{p}=10.01 \mathrm{~Pa} \cdot \mathrm{s}$, the retardation time $\lambda_{2}=\lambda_{1}\left(\eta_{s} / \eta_{p}\right)=0.001 \mathrm{~s}$, and a polymer shear modulus $G=\eta_{p} / \lambda_{1}=10 \mathrm{~Pa}$.

Before showing and discussing the nonlinear oscillatory response of the Giesekus model with these parameters, we first confirm the two "zero-limits" of oscillatory tests by showing the linear viscoelastic relaxation spectrum $\left(\gamma_{0} \rightarrow 0\right.$, all $\left.\omega\right)$ and the steady-shear flow curve $\left(\omega \rightarrow 0\right.$, all $\left.\gamma_{0}\right)$ in Fig. 4 . The crossover frequency of $1 / \lambda_{1}=1 \mathrm{rad} \cdot \mathrm{s}^{-1}$ is indicated in the linear relaxation spectrum and flow curve as a vertical dashed line. To the flow curve, we also add indications of the rate which is the inverse of the retardation time $1 / \lambda_{2}=1000 \mathrm{~s}^{-1}$ (vertical dashed line), and the zero-shear and infinite-shear viscosity predictions (angled dotted lines). It is important that we keep these responses in mind when analyzing the nonlinear oscillatory responses, as they represent the two extremes which the large-amplitude oscillatory tests will interpolate. In both the Chebyshev description of the SD constructions $\sigma^{\prime}$ and $\sigma^{\prime \prime}$ and FT rheology, only the linear regime frequency information is retained, while the information from the steady-shear flow curve is neglected. This contrasts with the SPP framework proposed by Rogers et al. (2011) where knowledge of both extremes is fully exploited, or elucidated, to gather physically meaningful material insights.

We now turn our attention to some representative calculated waveforms of the stress response of the Giesekus model to oscillatory shear of strain amplitude $\gamma_{0}=178$ and angular frequencies of $\omega=0.1,1$, and $10 \mathrm{rad} \cdot \mathrm{s}^{-1}$. These are shown from startup in Fig. 5 in color along with $\sigma^{\prime}$ as solid black lines. The steady-state responses correspond to the rotationally symmetric (of order 2 about the origin) orbits, while the startup response begins at the origin.

Following the approach of Ewoldt et al. (2008) for the $\omega=0.1 \mathrm{rad} \cdot \mathrm{s}^{-1}$ data, we obtain a positive first-order elastic (Chebyshev) coefficient $\left(e_{1}=0.0007 \mathrm{~Pa}\right)$, and a positive relative third-order elastic coefficient $\left(e_{3} / e_{1}=3.35\right)$ which could be interpreted as showing strain hardening. However, because the third harmonic is larger than the fundamental in this case, no such interpretation shall be ascribed. We also obtain a negative relative third-order viscous coefficient $\left(v_{3} / v_{1}=-0.29\right)$, which is interpreted in the scheme proposed by Ewoldt et al. (2008) as showing shear thinning (an interpretation supported by the form of the flow curve). Similar results are obtained for the two higher frequencies, where the first-order elastic coefficient is positive $\left[e_{1}=0.002 \mathrm{~Pa}\left(\omega=1 \mathrm{rad} \cdot \mathrm{s}^{-1}\right)\right.$ and $\left.0.045 \mathrm{~Pa}\left(\omega=10 \mathrm{rad} \cdot \mathrm{s}^{-1}\right)\right]$,
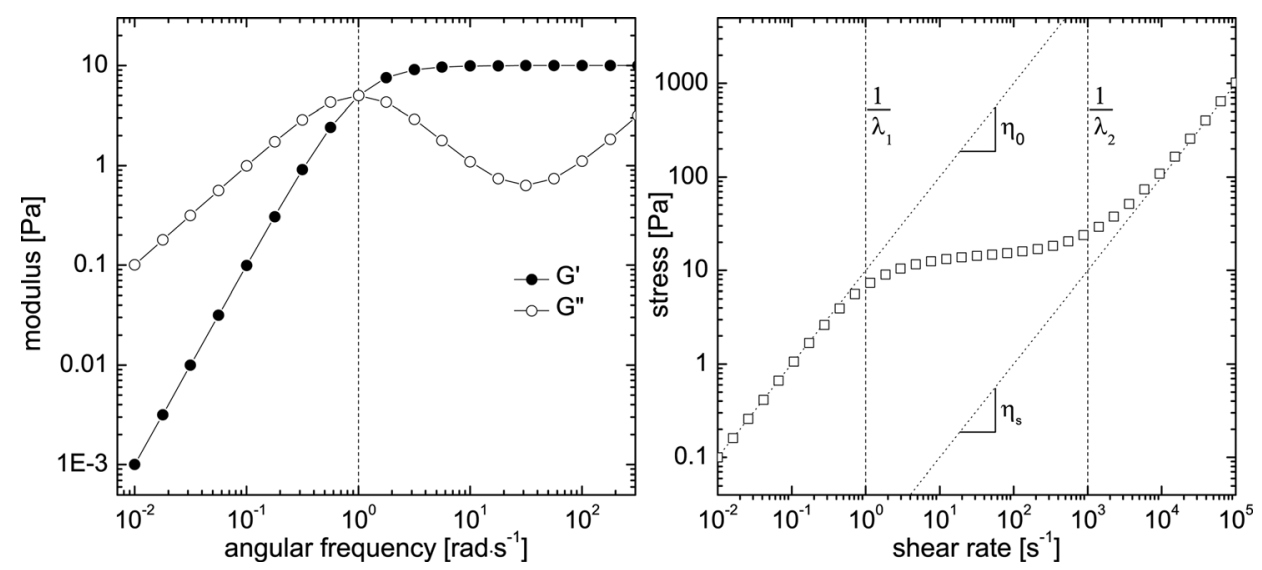

FIG. 4. Left-Linear viscoelastic relaxation spectrum of the Giesekus model showing plateau modulus of $10 \mathrm{~Pa}$ and a relaxation time of $1 \mathrm{~s}$. Right-Steady-shear flow curve showing predicted responses based on $\eta_{0}, \eta_{s}$ (angled dotted lines) and the inverses of the two relaxation times $\lambda_{1}$ and $\lambda_{2}$ (vertical dashed lines). 

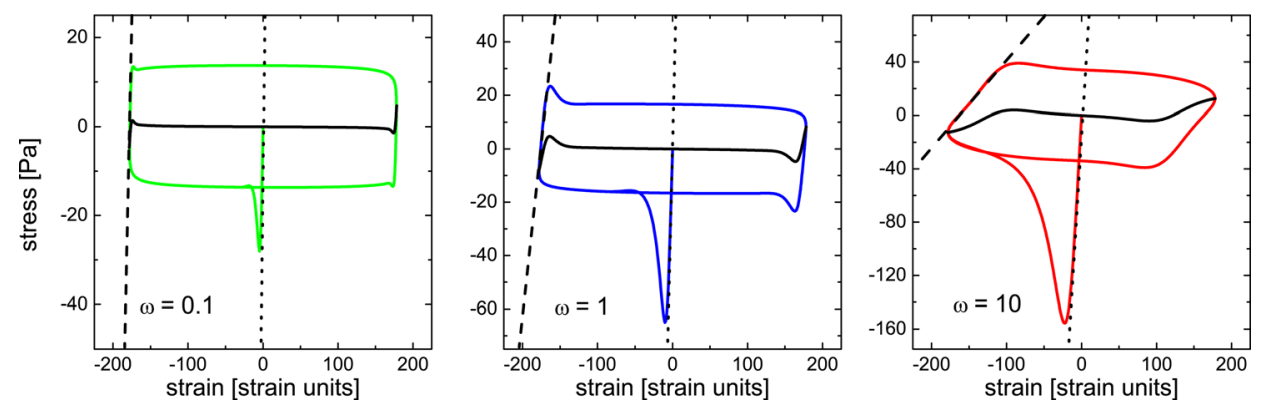

FIG. 5. Representative waveforms of the Giesekus model calculated under oscillatory shear conditions of $\gamma_{0}=178$ and $\omega=0.1$ (left), 1 (centre), and 10 (right) $\mathrm{rad} \cdot \mathrm{s}^{-1}$ displayed in elastic Lissajous-Bowditch curves. Colored lines show the calculated stress response from startup, which quickly becomes the "steady-state" response. Solid black lines indicate $\sigma^{\prime}$ and dashed and dotted black lines are described in the text.

which would be interpreted as indicating very weak, but increasing with frequency, elastic contributions. The potential for interpreting strain hardening behavior also changes with frequency as evidenced by relative third-order elastic (Chebyshev) coefficients $\left(e_{3} / e_{1}=5.13\right.$ at $\omega=1 \mathrm{rad} \cdot \mathrm{s}^{-1}$, where no interpretation is ascribed, and $e_{3} / e_{1}=0.80$ at $\omega=10 \mathrm{rad} \cdot \mathrm{s}^{-1}$, where the strain hardening interpretation is attached). For completeness, we note that the first-order viscous coefficients for $\omega=1$ and $10 \mathrm{rad} \cdot \mathrm{s}^{-1}$ are positive $\left(v_{1}=0.12 \mathrm{~Pa} \cdot \mathrm{s}\right.$ and $0.02 \mathrm{~Pa} \cdot \mathrm{s}$, respectively), which indicate a decreasing linear viscous contribution with increasing frequency, and relative third-order viscous coefficients $v_{3} / v_{1}=-0.29$ and 0.026 , which would be interpreted as indicating a change from shear thinning to shear thickening with increasing frequency.

We now interpret the responses of the Giesekus model in terms of the SPP framework used previously in the analysis of the Bingham and modified Bingham models. We have already seen that with the model parameters in use there is a polymer shear modulus of $10 \mathrm{~Pa}$ and that the flow curve has a plateau at approximately $10 \mathrm{~Pa}$ between rates of 1 and $1000 \mathrm{~s}^{-1}$. We propose that the waveforms observed in Fig. 5 are better understood as representing an elastic extension which yields and is followed by viscous flow. In support of this claim, we show in Fig. 5 straight lines (black dashed lines) of constant differential modulus $(\partial \sigma / \partial \gamma)$ superimposed on the steady-state orbits displayed as elastic LissajousBowditch curves. Interestingly, the value of modulus obtained in this manner (which is calculated at the point where the second derivative of the stress with respect to strain, which could be interpreted as the rate of softening, is closest to zero before the local stress maximum. That is, the value of the differential modulus we take is from the point where the first derivative of the stress with respect to the strain changes least prior to the stress overshoot) is a nonlinear function of frequency, as shown in Fig. 6. This nonlinear dependence on the frequency indicates that the elastic response does not reflect the linear modulus and can be rationalized by noting that the model contains a constant relaxation time, here set to $1 \mathrm{~s}$. It is also an important part of the rationalization that LAOS has a nontrivial shear history. In order to measure the linear modulus of $10 \mathrm{~Pa}$ in LAOS tests, we would expect that the frequency of shearing would have to be high enough in order for the model not to relax stress while being extended. The period of oscillation would also have to be much longer than the relaxation time in order for full relaxation to have taken place between yielding events so that the equilibrium state is probed. These, of course, are mutually conflicting ideas that result in moduli smaller than the linear modulus being calculated from LAOS responses. Even at an angular frequency of $\omega=0.1$ $\mathrm{rad} \cdot \mathrm{s}^{-1}=\omega \lambda_{1}=0.1$, the measured modulus is only $2 / 3$ of the linear viscoelastic value. 


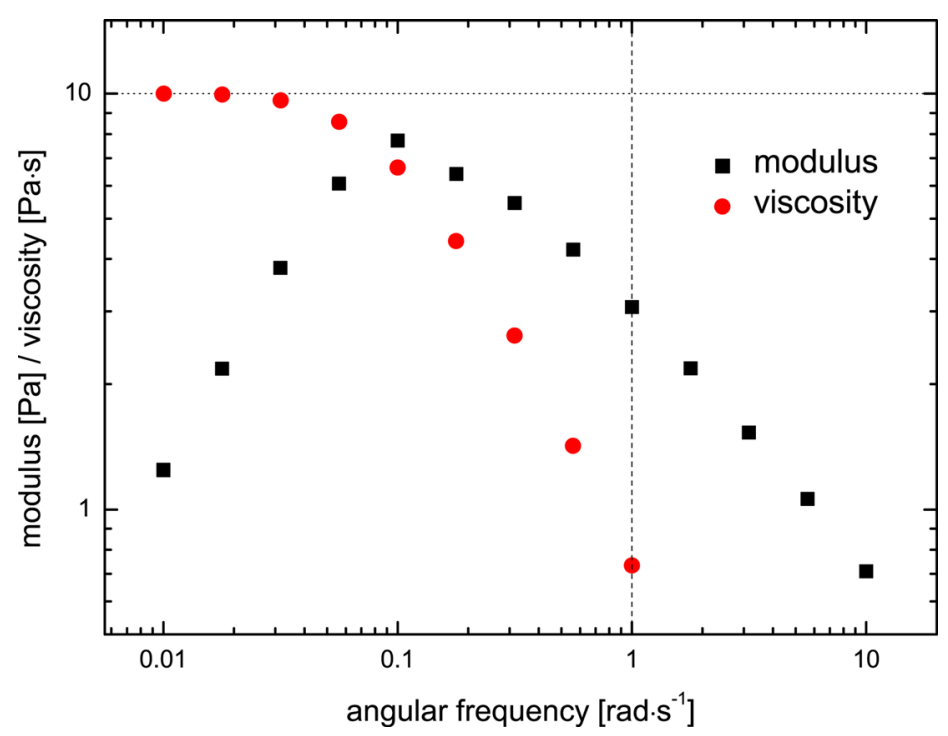

FIG. 6. The differential modulus and differential viscosity (see text) measured from calculated responses of the Giesekus model to a strain amplitude of 178, some of which are shown in Fig. 5. The polymer shear modulus of $10 \mathrm{~Pa}$, indicated by the horizontal dotted line, is never fully recovered in the frequency regime investigated, while the zero-rate viscosity of $10 \mathrm{~Pa} \cdot \mathrm{s}$ is achieved at low frequencies, as expected.

Below a frequency of $\omega \lambda=0.1$ (at an amplitude of $\gamma_{0}=178$ ), the (differential) modulus drops because the increased time taken to complete an oscillation is sufficient for the model to relax stresses (that is, behave like a fluid).

We also show in Fig. 6 the maximum instantaneous differential viscosity, which is calculated as the maximum value of the numerically differentiated stress with respect to rate (full waveforms are not shown as functions of rate). In the limit that the frequency approaches zero, that is as $\omega \rightarrow 0$, we expect to obtain the zero shear viscosity of $10.01 \mathrm{~Pa} \cdot \mathrm{s}$. We observe this occurring in Fig. 6 at frequencies lower than $\sim 0.03 \mathrm{rad} \cdot \mathrm{s}^{-1}$. At frequencies higher than about $0.1 \mathrm{rad} \cdot \mathrm{s}^{-1}$, the maximum instantaneous viscosity decreases rapidly. We associate this decrease in viscosity with the increasing frequency. This results in a decreased ability of the model to dissipate energy, resulting in a more solidlike response.

Our rationalization of the trend of a decreasing modulus with increasing frequency is further supported by observing not the steady-state response, but the transient response from startup (where the initial stress is zero), also shown in Fig. 5. Examining the initial stress response in this way removes all complications resulting from the shear history and we therefore expect to retain the polymer shear modulus. When this data is viewed, it becomes clear that the very first response of the system to LAOS is to deform elastically, where the stress is proportional to the strain, with the proportionality constant being the polymer shear modulus. This is marked by the coincidence of the stress response and the dotted black lines in Fig. 5. The width of the overshoot peak is related to the relaxation time $\left(\lambda_{1}=1 \mathrm{~s}\right)$ and therefore appears to be wider for higher frequencies (where $1 \mathrm{~s}$ makes up a larger fraction of the period of oscillation).

Prior to the stress overshoot, which we interpret as representing static yielding, the model responses display strain softening (a decrease in the differential modulus with increasing strain), which can be thought of as modeling plastic deformation. After the stress overshoot (i.e., yielding), the material flows as described by the flow curve. Directly after yielding, where the stress overshoots are observed in Fig. 5, the stress 
response of the system can be considered a convolution of the stress from the flow curve with the memory function. If the relaxation is fast compared with the frequency of oscillation, the memory of the system decays leaving just the stress response of the flow curve. This can be seen from the data of Fig. 7 where the oscillatory stress for the cases shown in Fig. 5 is superimposed on the steady-shear flow curve of Fig. 2. This method of display emphasizes the effects of a constant and finite relaxation time in that there is a nonzero stress at an instantaneous shear rate of zero. This effect arises whenever a fluidized material is sheared in an (sinusoidally) oscillatory manner: the material attempts to follow the flow curve, but the instantaneous shear rate is decreased faster than the material can relax (the rate of which is set by the relaxation time) the stress set by the flow curve. An examination of the linear viscoelastic response (in the linear regime, the stress residual in the system at zero rate is set by the storage modulus) of the Giesekus model [Bird et al. (1987)] suggests that the residual stress in the Giesekus model at an instantaneous shear rate of zero should be equal to $\left[\eta_{0}\left(\frac{\dot{\gamma}_{0} \omega\left(\lambda_{1}-\lambda_{2}\right)}{1+\lambda_{1}^{2} \omega^{2}}\right)\right]$. In direct analogy with the flow curve, which is a pictorial representation of the relation $\sigma=\eta \dot{\gamma}$, a plot of $\left.\sigma\right|_{\dot{\gamma}=0}$ vs $\left(\frac{\dot{\gamma}_{0} \omega\left(\lambda_{1}-\lambda_{2}\right)}{1+\lambda_{1}^{2} \omega^{2}}\right)$ should superimpose with the flow curve. In the case where $\lambda_{1} \gg \lambda_{2}$, and therefore $\left(\lambda_{1}-\lambda_{2}\right) \approx \lambda_{1}$ the result reduces to the same expression as for the linear Maxwell model. Such a plot can therefore act as an alternative method for determining $\lambda_{1}$. While this result is interesting, it does require prior knowledge of, or an assumption of the form of, the material constitutive equation to calculate. We reserve a full investigation of this topic for a further publication so as not to dilute the message of the current submission. It should be noted that the oscillatory data displayed in Fig. 7 that fall below the flow curve are from the elastic extension previously discussed and are shown merely for completeness.

The interpretation of the response of the Giesekus model offered here is very similar to that of the Bingham and modified Bingham models discussed previously. We suggest that greater physical understanding can be garnered by viewing the calculated response to LAOS as representing a sequence of purely elastic and viscous physical processes. We

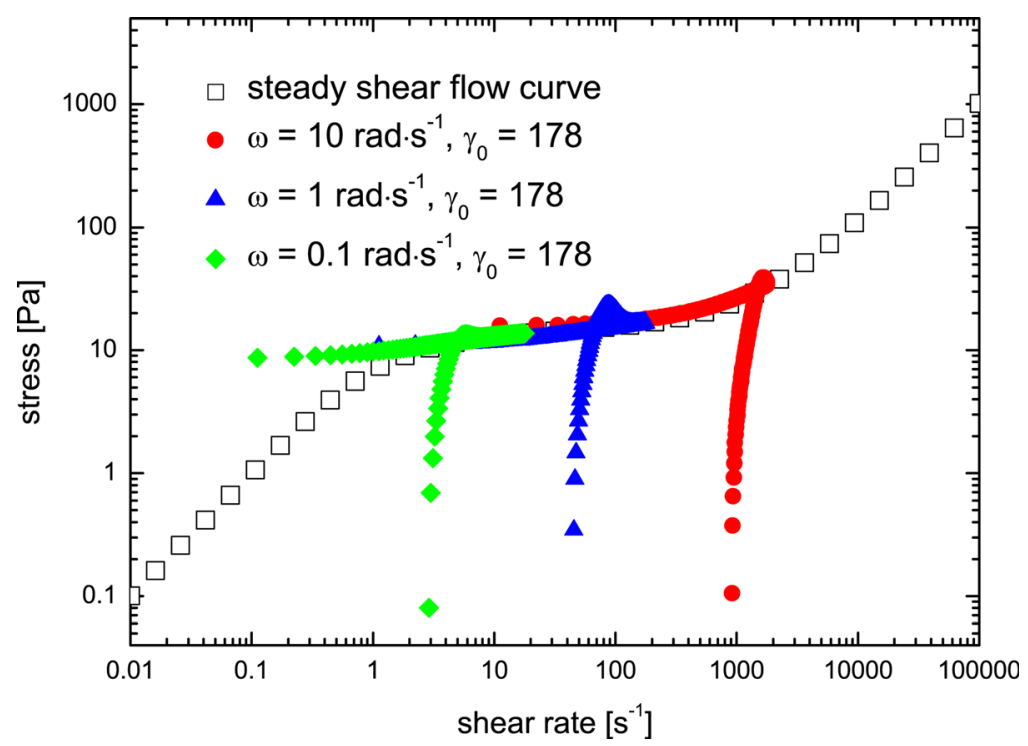

FIG. 7. Positive oscillatory stress (filled color symbols) superimposed on the steady-shear flow curve (unfilled squares). 
further suggest that the specific sequence of physical processes exhibited by the Giesekus model is of elastic extension, yielding, stress relaxation (at a rate set by the model relaxation time), followed by viscous flow (nearly identical to that of the steady-state response of the system indicated by the flow curve). Because of the differing natures of the effects of each one of these processes, it is sensible to analyze these calculated responses by a method other than a linear algebraic one. We must note, however, that similar to our discussions on the Bingham and modified Bingham models, the large rate dynamic viscosity as defined by Ewoldt et al. (2008), when applied to the LAOS response of the Giesekus model, will be equal to the steady-shear viscosity at the same shear rate, i.e., the rate amplitude. This is because, as shown in Fig. 7, the postyielding stress response is set by the flow curve. However, because the large-rate dynamic viscosity can only be accurately determined by Fourier or Chebyshev coefficients at a single point (the point of maximum shear rate), the utility of that approach is diminished when compared with the SPP method presented here.

\section{Discussion on the SD method and Chebyshev extension as applied to the examples given}

What causes the fundamental differences between the designed and calculated responses and the interpretations of $\sigma^{\prime}$ and $\sigma^{\prime \prime}$, and the $e_{n}$ 's and $v_{n}$ 's suggested by the Chebyshev extension of the SD method? The problem lies in the interpretation of the $\sigma^{\prime}$ and $\sigma^{\prime \prime}$ parameters. In the cases of the Bingham, the modified Bingham, and the Giesekus models presented here, for large parts of the stress response waveforms, highlighted in Fig. 8, $\sigma^{\prime}$ and $\sigma^{\prime \prime}$ represent conflations of strain-related stresses (the elastic stretching) and rate-related stresses (the viscous flow). It is this averaging over strain-dependent and rate-dependent regions, where different mechanisms are operating, that causes the discrepancies between the interpretations offered by the Chebyshev extension of the SD method and the true nature of the model response. These discrepancies, in turn, are effectively forced into $\sigma^{\prime}$ and $\sigma^{\prime \prime}$ by the stringent symmetry assumption that defines them. Nonlinear responses in general (in the Lissajous-Bowditch representation), only possess rotational symmetry of order 2 about the origin, making them (in a temporal representation) $\infty \times$ (step) frieze groups [Rogers and Vlassopoulos (2010)]. The symmetry assumptions of the definitions of $\sigma^{\prime}$ and $\sigma^{\prime \prime}$ [Eqs. (2) and (3)] are the same as those of linear responses, which (in the Lissajous-Bowditch representation) possess, in addition to rotational symmetry, two mirror planes (along the major and minor axes), which in a temporal representation makes them $2 * \infty$ (spinning sidle) frieze groups. This is the reason why $\sigma^{\prime}$ and $\sigma^{\prime \prime}$, as defined by Eqs. (2) and (3), can be used to determine $G^{\prime}$ and $G^{\prime \prime}$ in the linear regime [Ewoldt et al. (2008)]. One must therefore conclude that, in systems where the stress response represents a sequence of physical processes, such as the yielding examples presented here, any interpretation of the decomposed variables $\sigma^{\prime}$ and $\sigma^{\prime \prime}$, and any subsequent analysis upon which it is based has the potential to conflate information from elastic and viscous processes.

The arguments presented here in favor of approaches other than linear algebraic ones to the analysis of yielding responses to LAOS can be generalized to cases where the total stress response is the result of a sequence of physical processes. In the examples given here, that sequence is of an elastic process followed by a viscous one. The same arguments would also hold in the case where polymers are subjected to oscillatory shear that induces elastoplastic deformation after a short interval of linear elastic extension. Generally, the arguments relating to the conflation of $\sigma^{\prime}$ and $\sigma^{\prime \prime}$ and therefore of the misinterpretations of the $e_{n}$ 's and $v_{n}$ 's apply as long as there are different mechanisms responsible for different parts of the oscillatory stress response. 
(a)

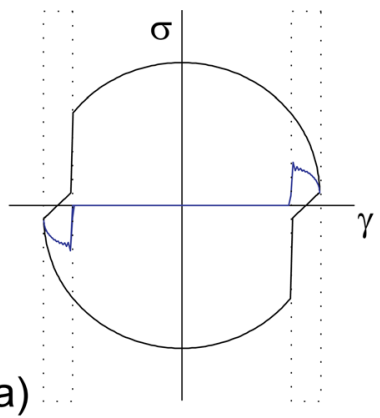

(d)

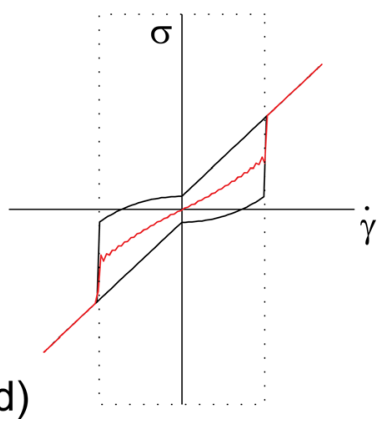

(b)

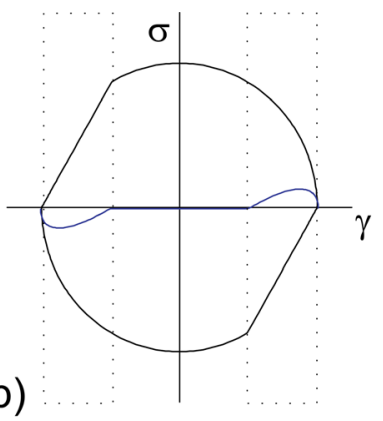

(c)

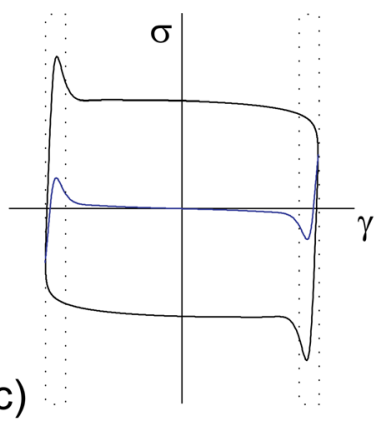

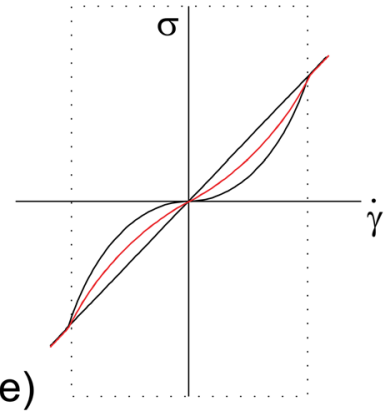

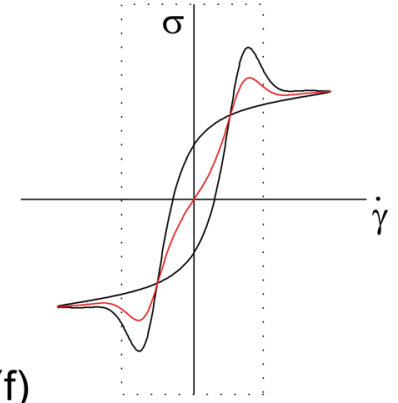

FIG. 8. Three representative waveforms [in black with $\sigma^{\prime}$ in blue $(\mathrm{a}-\mathrm{c})$ and $\sigma^{\prime \prime}$ in red $(\mathrm{d}-\mathrm{f})$ ] of responses that are best viewed as resulting from a sequence of physical processes: ( $a$ and $d$ ) The Bingham model, ( $b$ and e) the modified Bingham model (see text) and (c and f) the Giesekus model (response to $\gamma_{0}=178, \omega \lambda=1$ ). The portions in the dotted boxes show the parts of $\sigma^{\prime}$ and $\sigma^{\prime \prime}$ that conflate stresses from elastic and viscous mechanisms.

\section{E. A comment on the presentation methods of LAOS data}

All currently used analysis techniques assume a spatial symmetry of the response. The impact of such symmetry assumptions is that stress responses to LAOS need not be displayed as full Lissajous-Bowditch curves. A general stress response to LAOS exhibits a minimum of rotational symmetry of order 2 about the origin in both the elastic and viscous Lissajous-Bowditch representations [Rogers and Vlassopoulos (2010), stated in Cho et al. (2005) in the form of Eqs. (2) and (3)]. It is therefore thought to be redundant to show more than half of a waveform. Indeed, as was shown earlier, $\sigma^{\prime}$ and $\sigma^{\prime \prime}$ themselves can be calculated from only one half of the period. The natural presentation in the SPP formalism is to only show the positive-rate information. That is, one needs only to display the stress response from $-\gamma_{0}$ to $\gamma_{0}$ (and not the symmetric part that goes from $\gamma_{0}$ to $-\gamma_{0}$ ) in the elastic representation and the positive-rate stress response in the viscous representation.

Cho et al. (2005) and Ewoldt and McKinley (2010) displayed LAOS data as onedimensional closed traces imbedded in a three-dimensional space defined by the mutually orthogonal strain, strain-rate, and stress axes. In this representation, the SPP framework suggests that a time-dependent rotation of the axes should be implemented in order to obtain the best viewing of the data. In the cases examined here, where purely elastic responses give way to purely viscous responses, the three-dimensional space is best viewed initially normal to the stress-strain plane, i.e., in the elastic Lissajous-Bowditch representation. As the Bingham, modified Bingham, or Giesekus models yield in response to increasing strain, the axes are rotated by an angle of $-\pi / 2$ about the stress axis (positive rotations are defined to be in the anticlockwise direction as per the righthand rule when viewing from the positive stress axis) so that the viewer is then normal to 

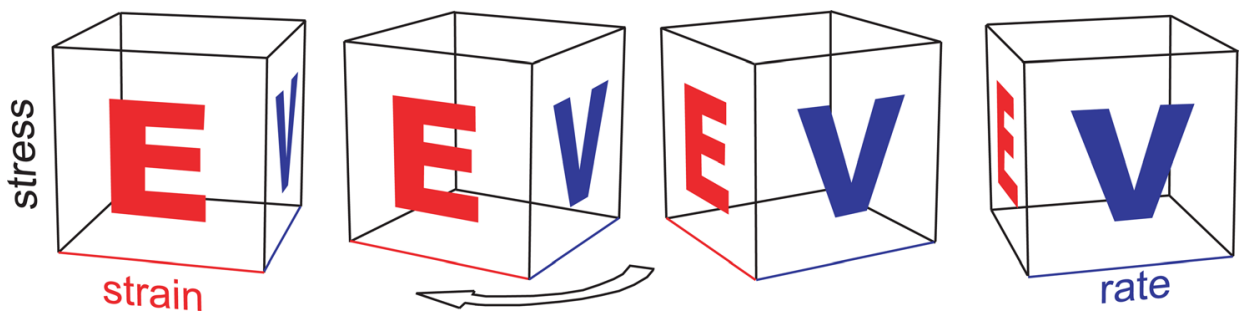

FIG. 9. LAOS data can be displayed as one-dimensional closed traces in a three-dimensional space defined by the strain (red), strain-rate (blue), and stress (black) axes. Rather than viewing these traces as fixed in time, the SPP framework suggests rotating the space from elastic (E) to viscous (V) presentations (left to right) as a function of time.

the stress-rate plane and views the response in the viscous Lissajous-Bowditch representation. The time taken for the rotation should be dictated in some way by the relaxation time of the model/material. In the case of the Bingham and modified Bingham models, the rotation should be applied instantaneously once the yield conditions have been exceeded as the model response changes instantly from elastic to viscous. In the case of the Giesekus model, the rotation should take place over an interval of time shorter than the relaxation time, $\lambda_{1}$. Rotations of $+\pi / 2$, which return the space to the initial configuration, should be applied when the stress falls below the (dynamic) yield stress. A visual guide to the three-dimensional space and the rotation scheme is displayed in Fig. 9, where the strain axis is shown in red, the strain-rate axis is blue, and the stress axis is black. The elastic representation is shown as a capital "E" and the viscous representation is shown as a capital "V." Time is assumed to progress from left to right.

The required rotations of the three model responses are indicated in Fig. 10. Figure 10(a) shows the Bingham model, Fig. 10(b) shows the modified Bingham, and Fig. 10(c) shows the response of the Giesekus model to $\gamma_{0}=178, \omega \lambda=1$. Projections onto the stress-strain (the elastic representation), stress-rate (the viscous representation), and strain-rate planes are also displayed. Under the SPP framework, one ought to view each of the three model responses for the red sections of the waveforms in Fig. 10 in the elastic representation, i.e., normal to the stress-strain plane. For the blue sections of the waveforms, the data are best viewed normal to the stress-rate plane in the viscous representation. Between these two limiting responses, the space needs to be rotated as displayed in Fig. 9.

The rotation concept also allows for an alternative interpretation of linear viscoelastic responses. In the linear regime, any stress response can be written as $\sigma_{0} \sin (\omega t+\delta)$
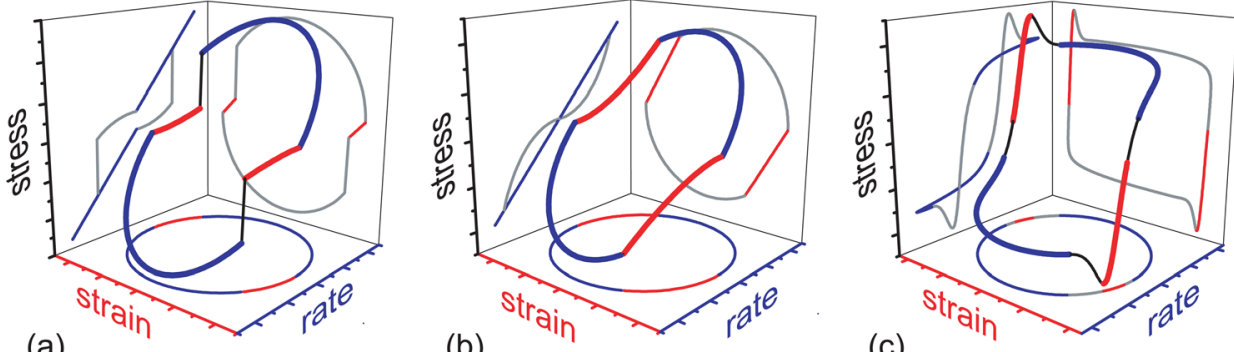

(c)

FIG. 10. The three models used in this work ( $\mathrm{a}-$ Bingham $/ \mathrm{b}-$ modified Bingham $/ \mathrm{c}$ - Giesekus response to $\left.\gamma_{0}=178, \omega \lambda=1\right)$ presented in the 3D space defined by the stress, strain, and shear rate axes. Projections onto the elastic (stress-strain) and viscous (stress-rate) planes previously presented in Figs. 2, 3, and 8 are also displayed. Under the SPP framework, the best viewing of this data includes a rotation as the responses change from elastic (red lines) to viscous (blue lines). 
[Ferry (1980)] where $\delta$ is referred to as the phase angle between the stress and strain vectors. An object in three dimensions can be rotated by an angle $\theta$ about the positive z-axis by the rotation matrix

$$
R_{z}(\theta)=\left[\begin{array}{ccc}
\cos \theta & -\sin \theta & 0 \\
\sin \theta & \cos \theta & 0 \\
0 & 0 & 1
\end{array}\right]
$$

By applying such rotations, it can be shown that for any linear viscoelastic stress response given by the column vector $\left[\begin{array}{lll}\gamma(t) & \dot{\gamma}(t) & \sigma(t)\end{array}\right]^{\mathrm{T}}$, there exists an angle of rotation $\theta$, such that no area is enclosed by the curve. Visual proof of this is shown in Fig. 11 where three different linear viscoelastic responses, corresponding to phase angles of $\delta=\pi / 8, \pi / 4$, and $3 \pi / 8$ are rotated by various angles $-\theta$, showing that their two-dimensional projections appear as straight lines when $\theta=\delta$.

Formal proof proceeds by calculating the area, $A^{\prime}$, of the projection of the curve onto the stress-strain plane for an arbitrary rotation angle $-\theta$. We use Green's theorem to equate the area integral with a line integral and note $A^{\prime}=\oint \sigma(t) d \gamma=\oint \sigma(t) \dot{\gamma}(t) d t$, where the form of $\dot{\gamma}(t)$ we take is the rotated rate vector $\dot{\gamma}^{\prime}(t)=\gamma(t) \sin (-\theta)+\dot{\gamma}(t) \cos (-\theta)$. Therefore

$$
\begin{aligned}
A^{\prime} & =\oint \sigma(t) \dot{\gamma}^{\prime}(t) d t \\
& =\int_{0}^{2 \pi / \omega} \sin (\omega t+\delta)[\sin (\omega t) \sin (-\theta)+\cos (\omega t) \cos (-\theta)] d t \\
& =\frac{\pi}{\omega}[\cos (\theta) \sin (\delta)-\sin (\theta) \cos (\delta)],
\end{aligned}
$$

which is identically equal to zero when $\theta=\delta$.

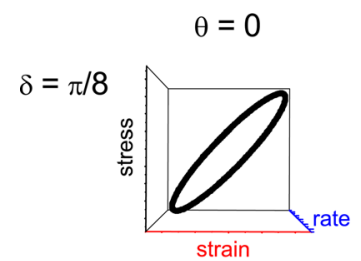

$$
\theta=\pi / 8
$$

$\theta=\pi / 4$

$$
\theta=3 \pi / 8
$$

$\delta=\pi / 4$
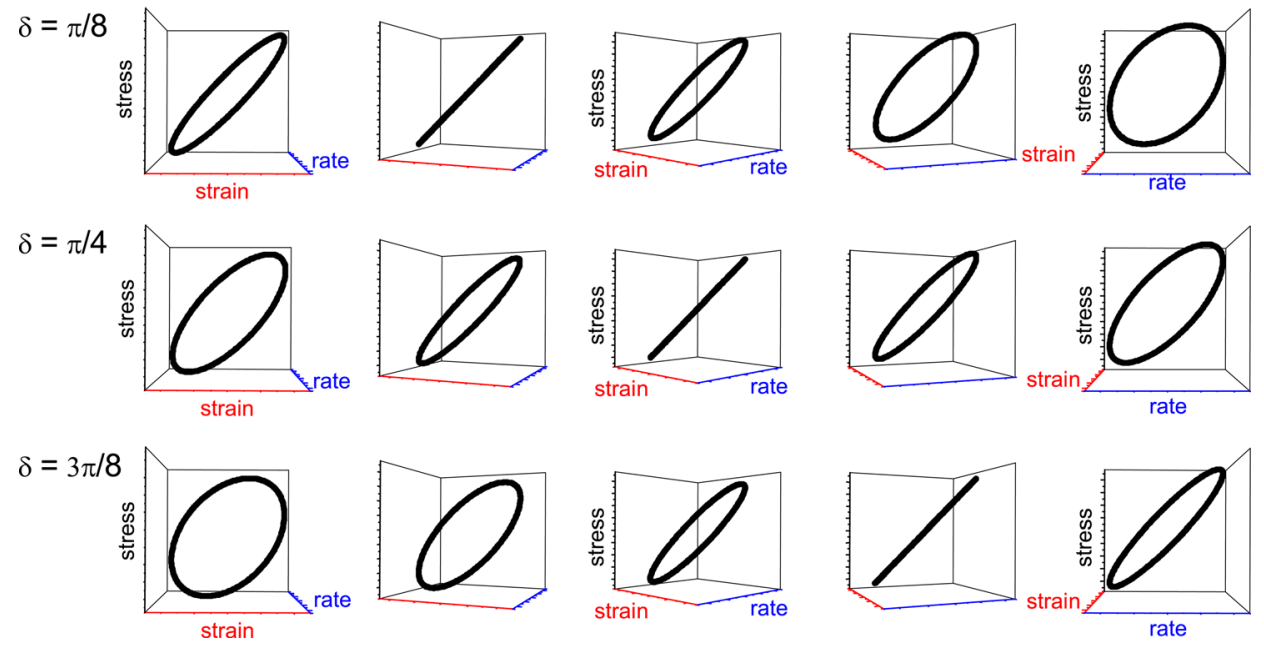

FIG. 11. Three linear viscoelastic stress responses, characterized by $\delta=\pi / 8$ (top row), $\pi / 4$ (middle row), and $3 \pi / 8$ (bottom row) are rotated by angles $-\theta$ as displayed at the top. No area is enclosed by the curves when the angle of rotation is equal to the phase angle of the response, i.e. when $\theta=\delta$. 
The existence of a rotation angle where zero area is enclosed by an arbitrary linear viscoelastic stress response naturally suggests a new measure of viscoelastic nonlinearity which we discuss in the Appendix.

\section{F. Comments on Fourier transformation as applied to LAOS responses}

Because of the equivalence relation between the Chebyshev and Fourier coefficients and the SD parameters as shown by Ewoldt et al. (2008) and reproduced here as Eqs. (1), (4), and (6), the arguments previously applied to the interpretation of the Chebyshev approach also apply to FT rheology. There is, however, a fundamental difference to the way FT rheology is used compared with the Chebyshev approach. While the Chebyshev approach describes the SD constructions $\sigma^{\prime}$ and $\sigma^{\prime \prime}$, FT rheology decomposes the full stress response. The use of FT rheology has, in many cases, been reduced to an examination of the relative intensity of the third harmonic. Furthermore, the relative intensity of the third harmonic is given elevated status and equated with "nonlinearity" [Hyun and Wilhelm (2009)]. This is certainly true when the only measureable higher harmonic is the third, which may be true in the MAOS regime as defined by Hyun and Wilhelm (2009). When it is clear that other harmonics are present in a response, they cannot be neglected; an integration of all higher harmonics, including phase information, would better represent a "degree of nonlinearity" (see Appendix).

Second, reconstructions do not necessarily constitute useful physical descriptions, especially when the fundamental assumptions leading to the reconstruction are false, e.g., the strict symmetries exhibited by the basis states. It is possible to fit a sinusoid with frequency equal to that of the imposed deformation to any periodic response. However, when that periodic response represents a sequence of physical processes, it is a misinterpretation to suggest that this sinusoid somehow represents a "linear response" of the model/material. In this vein, we agree with the interpretation suggested by McKinley and co-workers [Ewoldt et al. (2008)] that the first harmonic represents some sort of "average" measure over the entire cycle and contains information from both linear and nonlinear processes.

The final issue we wish to raise relates to an a priori knowledge of the frequency at which experimental noise occurs. In Figs. 5 and 7, the initial stress overshoot of the Giesekus model to excitation of $\gamma_{0}=178$, at $\omega=0.1 \mathrm{rad} \mathrm{s}^{-1}$ takes up a very small fraction of the total wave. This means that many high-frequency contributions must be taken into account in order to accurately reconstruct this particular waveform. Further, Rogers et al. (2011) discussed the link between the often-used technique of linking individual harmonics with specific physical processes and many real-world and model fluid responses when they showed that if the material studied behaves like a power-law fluid, then infinitely many higher harmonics must be included for a faithful reproduction. If one performs an experiment with no prior knowledge of the upper bounds of the material response frequency domain, and sets an arbitrary upper limit (say $n=15$ ) on the number of higher harmonics to be included in the reconstruction, then there exists a very real possibility of misrepresenting the true stress response or missing features altogether. If, on the other hand, the Fourier information is to be used by experimentalists to construct local measures, which we remind the reader can only be defined at a limited number of specific locations such as $\gamma=0$ and $\gamma_{0}$, then the highest number of harmonics possible should be used. While some such local measures can be defined by infinite sums of Fourier or Chebyshev coefficients [Ewoldt et al. (2008)], viewing the stress response as being the result of a sequence of physical processes provides a description of the full stress response at all points, with physically meaningful interpretations. 


\section{CONCLUDING REMARKS}

The trend in recent years has been to use linear algebraic periodic techniques (be it the Chebyshev series description of the SD parameters or FT rheology) to analyze stress responses to LAOS simply because they are the result of a periodic excitation. The currently used methods of analysis proposed by, Ewoldt et al. (2008) [based on the work of Cho et al. (2005)] and Wilhelm et al. (2002) both assume a linear algebraic approach; an implicit assumption is made that the infinite number of basis states exhibit potentially unrealistic symmetries and that the only analysis issue is to find their amplitude and possibly phase. In FT rheology, where the total stress response is described, the basis is assumed to be the infinite set of sinusoids with different frequencies and each harmonic has its own phase. In the Chebyshev series description of $\sigma^{\prime}$ and $\sigma^{\prime \prime}$, the basis is taken as the infinite set of Chebyshev functions of the first kind which have fixed phases of either zero or $\pi / 2$ depending on whether they are being used to describe $\sigma^{\prime}$ or $\sigma^{\prime \prime}$. Both approaches suffer the same crippling issue of a lack of physical interpretability of the infinite basis sets they assume. The work presented here offers a solution to this problem by assuming that only the same two fundamental material responses assumed in linear regime analysis, elasticity and viscosity, exist but allowing them to vary with time throughout an oscillation. We have shown that by making these more physically realistic assumptions, we are able to fully describe, at all points in time, the nonlinear yielding responses of three models. It has even been shown that the SPP approach provides the opportunity to completely determine the constitute equation of the simple Bingham model, while the best that FT-rheology and the Chebyshev approach can do is to describe each individual response curve with large numbers of physically meaningless parameters.

We have shown that the symmetry requirements placed on $\sigma^{\prime}$ and $\sigma^{\prime \prime}$ mean that they are more suited to analyzing linear regime oscillatory data than they are to nonlinear responses. In the nonlinear yielding examples given here, $\sigma^{\prime}$ and $\sigma^{\prime \prime}$ conflate information from elastic processes and viscous processes. While we agree with the spirit of the SD method, that by using the full material response the elastic and viscous contributions (note that only two contributions are assumed) can be elucidated, we have shown that the symmetry restrictions of the SD, as currently stated, are too strict. In order to relax the symmetry requirements further, one is reduced to observing the full material response as we have here.

Finally, we have proposed that nonlinear oscillatory yielding stress responses should be viewed as being the results of periodic sequences, not linear combinations, of physical processes. This means that basis states are not assumed to be exhibit particular strict symmetries but are allowed to wax and wane throughout an oscillation. In the Bingham, modified Bingham, and Giesekus models presented here, the viscous response is not symmetric in the way assumed by the FT, SD, or Chebyshev approaches, but rather only exists once the yield conditions have been exceeded. There is, therefore, an inherent inability in all linear algebraic approaches, because of the assumption of the constancy of the basis states, to describe such temporally varying responses. While these waveforms can be described and reconstructed to arbitrary degrees of accuracy by both FT rheology and the Chebyshev approach, we propose that by viewing the responses to LAOS as being the results of sequences of physical processes, we are able to link all nonlinear oscillatory data to both the linear oscillatory regime and the steady shear flow curves. No other analysis technique provides this link. This link is invertible, meaning that if the linear regime response or flow curve is not known, that an SPP analysis of nonlinear LAOS responses can provide a way for at least partial elucidation within the experimental (frequency and shear rate amplitude) parameters. By taking this more general physically- 
rather than mathematically minded approach-we can better determine the processes which govern the behavior of the materials we study and obtain a greater and more complete understanding.

\section{APPENDIX}

We wish to define a new nonlinear parameter here, the interpretation of which better reflects the full nonlinear response to LAOS. Preliminary investigations suggest this new parameter is at least as sensitive to nonlinearities as Fourier transformation, and makes no assumptions about the continual presence, or form, of basis states.

We showed in Sec. II.5 that there exists an angle of rotation $\theta=\delta$ such that when a linear viscoelastic response, given by $\sin (\omega t+\delta)$, is rotated around the z-axis by $-\theta$, the $2 \mathrm{D}$ projection encloses no area (where the variable $\delta$ is the phase angle difference between the stress and strain vectors and is ill-defined for nonsinusoidal, nonlinear responses). This suggests that no such angle exists for nonlinear responses and that the best that can be done is a minimum area when rotated by some angle. We therefore define a new viscoelastic nonlinearity parameter as being the pair of numbers $\left[A_{\min }^{\prime}, \theta_{c}\right]$ given by the minimum unsigned area enclosed by the projection of any normalized stress response onto the normalized stress-strain plane under rotations of $0 \leq \theta \leq \pi / 2$ about the z-axis, which we denote as $A_{\text {min }}^{\prime}$, and the rotation angle, $\theta_{c}$, at which this minimum is achieved. This measure therefore gives a deviation from linear behavior (when $A^{\prime}{ }_{\min }>0$ ) and an angle, which can be thought of as a generalized phase difference, which characterizes whether the total stress response is predominantly elastic $\left(\theta_{c}<\pi / 4\right)$ or viscous $\left(\theta_{c}>\pi / 4\right)$. The benefit of this approach over FT-rheology, where one might be tempted to use the phase of the first harmonic, is that no assumption is made in this case with regards to the constant presence of basis states. No assumptions are made about the form (sinusoidal, Chebyshev, Legendre, Lebesgue, etc.) of the basis states either. This measure therefore better reflects the average phase of the entire response, taking into account the waxing and waning of the fundamental elastic and viscous material states. If one uses normalized stress, strain and rate inputs, the minimum unsigned area, $A_{\text {min }}^{\prime}$ can vary between 0 and 4 (though is typically on the order of 0.4 ), and the angle $\theta_{\mathrm{c}}$ may vary between 0 and $\pi / 2$. It should be noted that in most cases, the angle $\theta_{c}$ will be close to, but not equal to, the phase of the first harmonic. While the phase of the first harmonic is interpreted as representing the phase of the averaged response (which is itself assumed to be composed in some way of a combination of linear and nonlinear effects), the phase of this new parameter represents the phase of the total integrated nonlinear response.

In general, the projections of nonlinear responses will not entirely be positively oriented (where positive orientation is defined by the right-hand rule) nor will they be simple closed curves; they will contain alternating regions of positive and negative orientation and will intersect themselves multiple times. As a result, the nonlinear parameter must be defined by the minimum unsigned projected area. The unsigned area is obtained by subtracting the area of negatively oriented portions of the projected curves from the area enclosed by positively oriented regions. In practice, this must be carried out numerically.

This new measure combines the effects of all sources of nonlinearity, not simply those at a specific frequency, which makes it akin, in the FT rheology framework, to the integral of all higher harmonics, when the phases of individual harmonics are taken into account. Because all the available waveform information is used, this new measure provides more detail than the phase and intensity of the third harmonic, as used by Vittorias et al. (2007), and more information than the Q parameter proposed by Hyun and Wilhelm (2009), which 

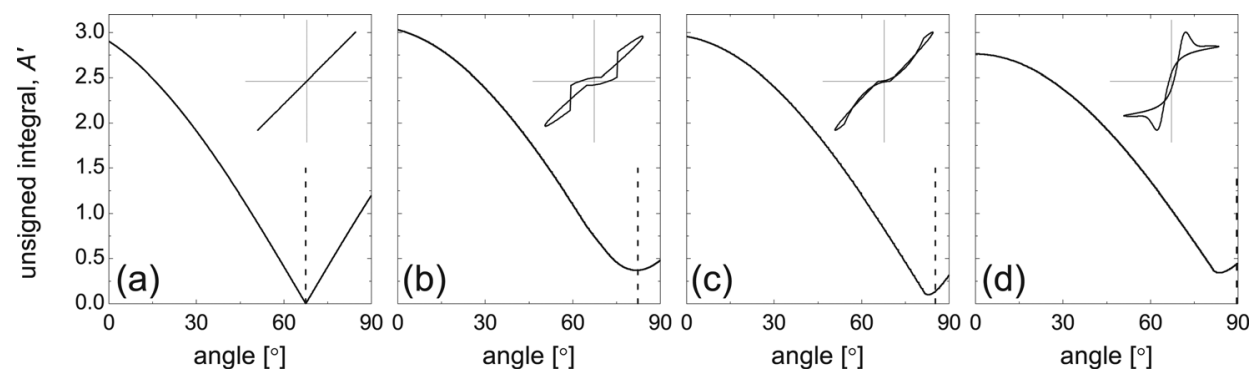

FIG. 12. The unsigned integral as a function of rotation angle for four normalized responses. (a) Linear response defined by $\sin (\omega t+3 \pi / 8$ ), (b) the Bingham model, (c) the modified Bingham model, and (d) the Giesekus model. Vertical dashed lines indicate the angular value of $\delta_{1}$, the phase of the first harmonic. The insets display the projections where minimum area is enclosed.

contains no phase information. The greatest benefit of this new parameter comes from the interpretability of the both components. Unlike the normalized intensity and phase of the third harmonic, the intensity and angle of the new parameter have direct and easily visualized interpretations.

We note that the greatest weakness of this new parameter is that in the low-frequency limit, purely viscous nonlinearities can ret urn a minimum unsigned area of zero at a rotation angle of $\pi / 2$. In this respect, the new parameter is not a general LAOS nonlinear parameter, but rather a viscoelastic nonlinearity parameter.

We show in Fig. 12 the unsigned area as a function of rotation angle for four normalized responses; (a) a linear response described by $\sin (\omega t+3 \pi / 8)$, (b) the Bingham model, (c) the modified Bingham, and (d) the Giesekus model when subjected to oscillatory shear conditions previously discussed. We show for comparison the phase of the first harmonic as vertical dashed lines for each response curve. We also show as insets in Fig. 12 the projections of each response when rotated by an angle $\theta_{\mathrm{c}}$, such that a minimum area is enclosed. From the positions of the minima plotted in Fig. 12, we can define the nonlinear parameters for each of the responses as being (phase of the first harmonic in parentheses)

$$
\begin{array}{ll}
\text { Linear }-\left[0,67.5^{\circ}=3 \pi / 8 \mathrm{rad}\right] & \left\{\delta_{1}=67.50^{\circ}\right\} \\
\text { Bingham }-\left[0.37,81.85^{\circ}\right] & \left\{\delta_{1}=82.37^{\circ}\right\} \\
\text { Modified Bingham }-\left[0.09,82.80^{\circ}\right] & \left\{\delta_{1}=85.26^{\circ}\right\} \\
\text { Giesekus }-\left[0.34,83.42^{\circ}\right] & \left\{\delta_{1}=89.57^{\circ}\right\}
\end{array}
$$

The information displayed in Fig. 12 can be used not only to calculate the new nonlinearity parameter but also to calculate the perfect plastic dissipation ratio $\phi$, defined by Ewoldt et al. (2010). The perfect plastic dissipation ratio is defined as being the ratio of areas enclosed by an arbitrary response in the stress-strain plane and a perfectly plastic response of the same stress and strain amplitudes. The ratio $\phi$ can be obtained by dividing the value of the unsigned integral at zero rotation by $4: \phi=A^{\prime}(0) / 4$.

\section{References}

Bird, R., R. Armstrong, and O. Hassager, Dynamics of Polymeric Liquids: Volume 1 Fluid Mechanics (Wiley, New York, 1987).

Cho, K. S., K. H. Ahn, and S. J. Lee, "A geometrical interpretation of large amplitude oscillatory shear response,” J. Rheol. 49, 747-758 (2005). 
Cho, K. S., K.-W. Song, and G.-S. Chang, "Scaling relations in nonlinear viscoelastic behavior of aqueous PEO solutions under large amplitude oscillatory shear flow," J. Rheol. 54(1), 27-63 (2010).

Dodge, J. S., and I. M. Krieger, “Oscillatory shear of nonlinear fluids I. Preliminary investigation,” Trans. Soc. Rheol. 15, 589-601 (1971).

Ewoldt, R., A. Hosoi, and G. McKinley, "New measures for characterizing nonlinear viscoelasticity in large amplitude oscillatory shear,” J. Rheol. 52(6), 1427-1458 (2008).

Ewoldt, R. H., and G. H. McKinley, "On secondary loops in LAOS via self-intersection of Lissajous-Bowditch curves,” Rheol. Acta 49, 213-219 (2010).

Ewoldt, R. H., P. Winter, J. Maxey, and G. H. McKinley, "Large amplitude oscillatory shear of pseudoplastic and elastoviscoplastic materials," Rheol. Acta 49, 191-212 (2010).

Ferry, J. D., Viscoelastic Properties of Polymers (Wiley, New York, 1980).

Harris, J., and K. Bogie, "The experimental analysis of non-linear waves in mechanical systems," Rheol. Acta. 6(1), 3-5 (1967).

Herschel, W. H., and R. Bulkley, "Konsistenzmessungen von Gummi-Benzollosungen," Kolloid-Z. 39, 291-300 (1926).

Hyun, K., and M. Wilhelm, "Establishing a new mechanical nonlinear coefficient Q from FT-rheology: First investigation of entangled linear and comb polymer model systems," Macromolecules 42, 411-422 (2009).

Klein, C., H. W. Spiess, A. Calin, C. Balan, and M. Wilhelm, "Separation of the nonlinear oscillatory response into a superposition of linear, strain hardening, strain softening and wall slip response," Macromolecules 40, 4250-4259 (2007).

Philippoff, W., "Vibrational measurements with large amplitudes," Trans. Soc. Rheol. 10, 317-334 (1966).

Renou, F., J. Stellbrink, and G. Petekidis, "Yielding processes in a colloidal glass of soft star-like micelles under large amplitude oscillatory shear (LAOS)," J. Rheol. 54(6), 1219-1242 (2010).

Rogers, S., and D. Vlassopoulos, "Frieze group analysis of asymmetric response to large amplitude oscillatory shear," J. Rheol. 54(4), 859-880 (2010).

Rogers, S. A., B. M. Erwin, D. Vlassopoulos, and M. Cloitre, "A sequence of physical processes determined and quantified in LAOS: Application to a yield stress fluid," J. Rheol. 55, 435 (2011).

Tee, T. T., and J. M. Dealy, "Nonlinear viscoelasticity of polymer melts," J. Rheol. 19, 595-615 (1975).

Vittorias, I., M. Parkinson, K. Klimke, B. Debbaut, and M. Wilhelm, "Detection and quantification of industrial polyethylene branching topologies via Fourier-transform rheology, NMR and simulation using the Pompom model," Rheol. Acta 46(3), 321 (2007).

Wilhelm, M., "Fourier-Transform rheology," Macromol. Mater. Eng. 287, 83-105 (2002).

Yoshimura, A. S., and R. K. Prud'homme, "Response of an elastic Bingham fluid to oscillatory shear," Rheol. Acta 26(5), 428-436 (1987).

Yu, W., P. Weng, and C. Zhou, "General stress decomposition in nonlinear oscillatory shear flow," J. Rheol. 53(1), 215-238 (2009). 